\title{
Dynamic effects of embedded macro-fiber composite actuators on ultra-light flexible structures of repeated pattern- a homogenization approach
}

\author{
A. Salehian ${ }^{\mathrm{a}, *}$ and T.M. Seigler ${ }^{\mathrm{b}}$ \\ ${ }^{a}$ Department of Mechanical and Mechatronics Engineering, University of Walterloo, Waterloo, Ontario, Canada \\ $N 2 L 3 G 1$ \\ ${ }^{\mathrm{b}}$ Department of Mechanical Engineering, University of Kentucky, Lexington, KY, USA
}

Received 11 February 2010

Revised 22 July 2010

\begin{abstract}
Motivated by deployable satellite technology, this article presents a homogenization model of an inflatable, rigidized lattice structure with distributed macro-fiber composite (MFC) actuation. The model is based upon a general expression for the strain and kinetic energy of a fundamental repeated element of the structure. These expressions are reduced in order and expressed in terms of the strain and displacement components of an equivalent one-dimensional vibration model. The resulting model is used to analyze changes in the structural natural frequencies introduced by the local effects of the added macro-fiber composite actuators for several configurations. A finite element solution is used as a comparison for the homogenization model, and the two are shown to be in good agreement, although the latter requires significantly less computational effort.
\end{abstract}

\section{Nomenclature}

$$
\begin{array}{ll}
A_{L}, A_{d}, A_{b} & =\text { cross-sectional area of longerons, diagonals and battens } \\
A^{(k)} & =\text { cross-sectional area of member } k \\
A_{M F C} & =\text { cross-sectional area of } M F C \\
m_{1}, m_{2} & =\text { coefficients of expansion of element strain energy } \\
n_{L} & =\text { number of actuators at cross section of the longeron } \\
n_{d} & =\text { number of actuators at cross section of diagonal } \\
C_{1}, C_{2}, C_{3}, & =\text { coefficients of expansion of truss element strain energy } \\
C_{4}, C_{5}, C_{6} & \\
E_{L}, E_{d}, E_{b} & =\text { modulus of elasticity of longerons, diagonals and battens } \\
E^{(k)} & =\text { modulus of elasticity of member } k \\
E_{M F C} & =\text { modulus of elasticity of } M F C \\
f & =\text { frequency (rad/sec) } \\
k & =\text { element number } \\
K_{L}, K_{d}, K_{b} & =\text { stiffness of longerons, diagonals and battens } \\
K_{L}^{*} & =\text { stiffness of actuated length of longeron }
\end{array}
$$

${ }^{*}$ Corresponding author. Tel.: +1 519888 4567, Ext. 38531; Fax: +1 519888 4333; E-mail: salehian@uwaterloo.ca. 


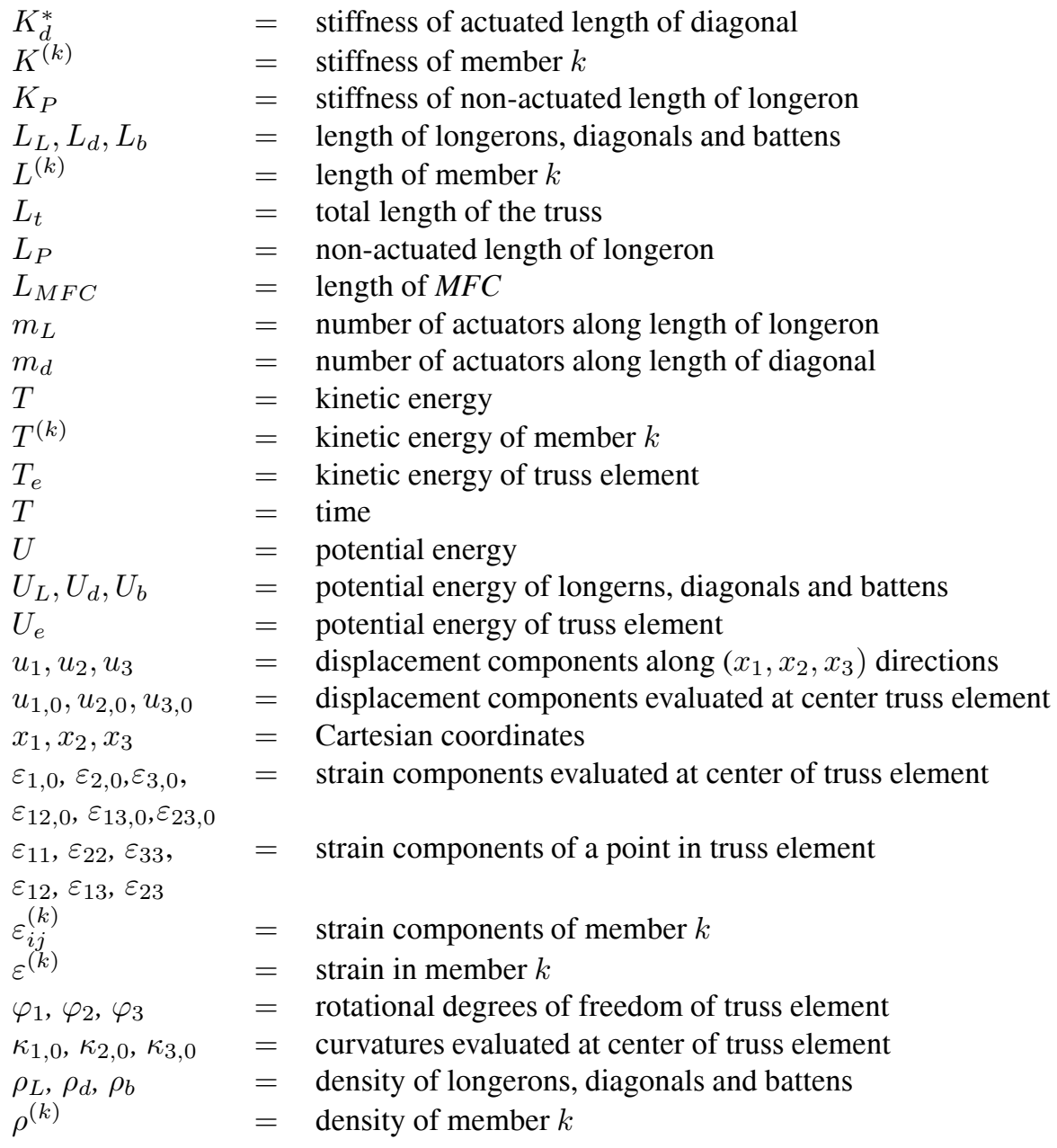

\section{Introduction}

We consider the vibration analysis of an inflatable satellite, which deploys and rigidizes in the form of a large truss structure. This type of deployable satellite belongs to a class of space-inflatable structures, or gossamer structures, characterized primarily by a low weight and packing volume. This technology enables placing larger metrology systems in space which results in improved coverage of antenna systems and enables them to keep track of smaller moving objects on Earth. A broad collection of research and development efforts in gossamer technology can be found in the two volume series edited by Jenkins [1,2], and the review articles of Ruggiero and Inman [3] and Ruggiero et al. [4].

Due to the large size of inflatable structures, significant time is required for their FEA vibration analysis. On the other hand, analysis of such structures can be significantly simplified, relative to the computational efforts required of finite discretization methods, by a process generally referred to as continuum modeling, whereby an equivalent beam or plate model is derived from careful assumptions relating the overall geometry and local strain properties of the equivalent continuum model and the truss structure. This simplified modeling technique is particularly attractive for control applications. As discussed in Noor et al. [5,6] and Salehian et al. [7-9], there are a few ways of going about this discrete to continuum transformation process [10-17]. The method of interest here, developed by Noor et al. [5], involves equating kinetic and potential energy expressions of the lattice and its intended beam equivalent. From these expressions and by making necessary assumptions for local free deformations, the equivalent beam-like equations of motion can be derived $[7,8]$. In addition to the computational savings involved with the continuum 
modeling approach, also the mathematical structure of the equations of motion is preserved in that is possible to directly relate changes in structural design (i.e., geometry, material properties, etc.) to changes in the vibration response. As satellites are subject to various sources of disturbance (e.g. repositioning, temperature changes, etc.), vibration mitigation is important feature of the structural design. The macro-fiber composite (MFC) actuator, a new generation of the piezoelectric actuators, is generally considered ideal for this purpose owing to its capacity for large force and displacement, and especially the ability to conform to curved structures. These actuators are essentially piezo-ceramic fibers and interdigitated electrodes embedded in a Kapton membrane.

The MFC has been considered in several recent studies for gossamer structures [18-22]: Park et al. [18] and Sodano et al. [19] demonstrated the effectiveness of the MFC as an actuator and sensor, respectively, for an inflatable torus; Jha and Inman [20] demonstrated sliding mode vibration control of the same structure; Tarazaga et al. [21] demonstrated vibration control of a space-rigidizable deployable composite boom using positive position feedback; in modeling efforts, Renno and Inman [22] derived the dynamic response for a MFC-actuated membrane strip.

In this article we apply this dynamic modeling approach to the aforementioned inflatable satellite truss structure motivated by the Innovative Space-based radar Antenna Technology (ISAT), a DARPA program to place a large antenna in the Medium Earth Orbit (MEO). We derive the homogenized equations of motion for a satellite truss structure with a distributed array of macro-fiber composite (MFC) actuators, thereby extending the authors' previous work $[7,8]$. The resulting model is used to analyze the vibration response of the composite satellite structure and to quantify the dynamic effects of the MFC actuators.

\section{Modeling}

\subsection{Strain energy}

In this section, the equations of motion are derived for an inflatable antenna truss with integrated MFC actuators. A single element of an inflatable antenna truss by ILC Dover is shown in Fig. 1. This element consists of battens, longerons and diagonal members as shown in the figure. The truss element used in this study is very similar to the figure shown except for that the diagonals have a symmetric pattern in Fig. 2 which is motivated by ISAT structure. The truss structure is made of this element and is the support for the ISAT satellite antenna.

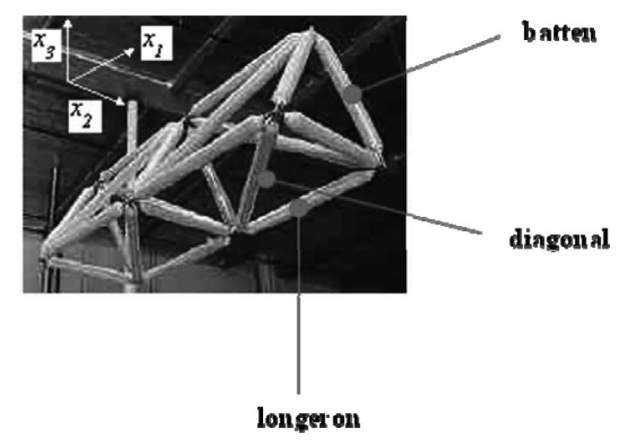

Fig. 1. Fundamental truss element of an inflatable truss (curtsey of ILC-Dover).

The general equations of strain are first derived by assumptions on the displacement field. The added stiffness of the MFC actuators is then evaluated and integrated into the strain energy expressions. Hamilton's principle is then applied to derive the partial differential equations of motion similar to [7,8].

To derive the kinetic and strain energy expressions for the resultant continuum, certain assumptions for the displacement components within an element are made. The individual members of the truss are modeled as bars, and the connections by pin joints. This results in a linearly varying displacement field where all the second order displacement components (bending and nonlinear terms) are neglected. Therefore, the three displacement components $\left(u_{1}, u_{2}\right.$ and $\left.u_{3}\right)$ along the coordinates $\left(x_{1}, x_{2}, x_{3}\right)$ shown in Fig. 2 can be written as [5,7,8]: 


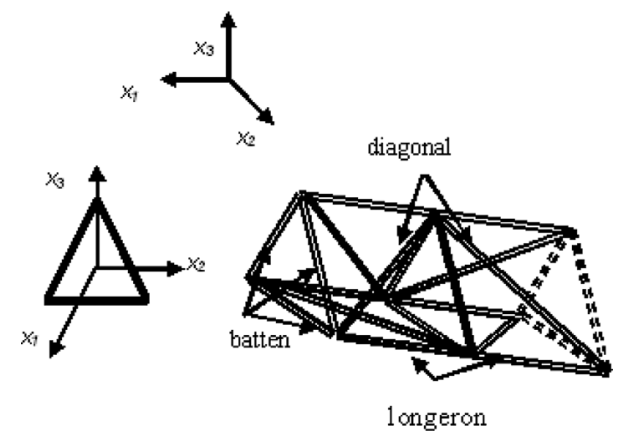

Fig. 2. Fundamental element of the ISAT truss.

$$
\begin{aligned}
& u_{1}\left(x_{1}, x_{2}, x_{3}\right)=u_{1,0}\left(x_{1}\right)-x_{2} \phi_{3}\left(x_{1}\right)+x_{3} \phi_{2}\left(x_{1}\right) \\
& u_{2}\left(x_{1}, x_{2}, x_{3}\right)=u_{2,0}\left(x_{1}\right)+x_{2} \varepsilon_{2,0}\left(x_{1}\right)+x_{3}\left[-\phi_{1}\left(x_{1}\right)+\frac{1}{2}\left(2 \varepsilon_{23,0}\left(x_{1}\right)\right)\right] \\
& u_{3}\left(x_{1}, x_{2}, x_{3}\right)=u_{3,0}\left(x_{1}\right)+x_{2}\left[\phi_{1}\left(x_{1}\right)+\frac{1}{2}\left(2 \varepsilon_{23,0}\left(x_{1}\right)\right)\right]+x_{3} \varepsilon_{3,0}\left(x_{1}\right)
\end{aligned}
$$

where, $u_{1,0}, u_{2,0}$ and $u_{3,0}$ are the displacement components at the center of a cross-section located at coordinate $x_{1}$ and $\varepsilon_{2,0}, \varepsilon_{3,0}$ and $\varepsilon_{23,0}$ are the extensional and shear strain components evaluated at the center of that cross section respectively. Also, $\phi_{1}, \phi_{2}$ and $\phi_{3}$ are the rotational components of the cross section around $x_{1}, x_{2}$ and $x_{3}$. For example, the displacement along $x_{1}$ coordinate for a point at a cross section located at $x_{1}$ is denoted as $u_{1}$. This can be found as the summation of the displacement $u_{1,0}$ at the center of the cross section and the projection of the components of the rotational degrees of freedom. Having the displacement field in Eq. (1), one can easily obtain the spatial derivatives of the displacement components to find the strain values $\varepsilon_{11}, \varepsilon_{22}, \varepsilon_{33}, \varepsilon_{12}, \varepsilon_{13}$ and $\varepsilon_{23}$. These strain components are considered the extensional and shear strain components and can be obtained at every node in the truss element. Equation (A.1) in Appendix-A presents a complete derivation for these strain components. A Taylor series expansion around the $x_{1}$ coordinate is considered to account for variations of strain components within an element. Finally, the strain components take the form:

$$
\begin{aligned}
\varepsilon_{11}= & \varepsilon_{1,0}-x_{2} \kappa_{2,0}+x_{3} \kappa_{3,0}+x_{1}\left(\frac{\partial \varepsilon_{1,0}}{\partial x_{1}}-x_{2} \frac{\partial \kappa_{2,0}}{\partial x_{1}}+x_{3} \frac{\partial \kappa_{3,0}}{\partial x_{1}}\right) \\
\varepsilon_{22}= & \varepsilon_{2,0}+x_{1} \frac{\partial \varepsilon_{2,0}}{\partial x_{1}}+\frac{1}{2}\left(x_{1}\right)^{2} \frac{\partial^{2} \varepsilon_{2,0}}{\partial x_{1}^{2}} \\
\varepsilon_{33}= & \varepsilon_{3,0}+x_{1} \frac{\partial \varepsilon_{3,0}}{\partial x_{1}}+\frac{1}{2}\left(x_{1}\right)^{2} \frac{\partial^{2} \varepsilon_{3,0}}{\partial x_{1}^{2}} \\
\varepsilon_{12}= & \frac{1}{2}\left(2 \varepsilon_{12,0}+x_{2} \frac{\partial \varepsilon_{2,0}}{\partial x_{1}}+x_{3}\left[-\kappa_{1,0}+\frac{1}{2}\left(2 \frac{\partial \varepsilon_{23,0}}{\partial x_{1}}\right)\right]\right. \\
& \left.+x_{1}\left(2 \frac{\partial \varepsilon_{12,0}}{\partial x_{1}}+x_{2} \frac{\partial^{2} \varepsilon_{2,0}}{\partial x_{1}^{2}}+x_{3}\left[-\frac{\partial \kappa_{1,0}}{\partial x_{1}}+\frac{1}{2}\left(2 \frac{\partial^{2} \varepsilon_{23,0}}{\partial x_{1}^{2}}\right)\right]\right)\right) \\
\varepsilon_{13}= & \frac{1}{2}\left(2 \varepsilon_{13,0}+x_{2}\left[\kappa_{1,0}+\frac{1}{2}\left(2 \frac{\partial \varepsilon_{23,0}}{\partial x_{1}}\right)\right]+x_{3} \frac{\partial \varepsilon_{3,0}}{\partial x_{1}}\right. \\
& \left.+x_{1}\left(2 \frac{\partial \varepsilon_{13,0}}{\partial x_{1}}+x_{2}\left[\frac{\partial \kappa_{1,0}}{\partial x_{1}}+\frac{1}{2}\left(2 \frac{\partial^{2} \varepsilon_{23,0}}{\partial x_{1}^{2}}\right)\right]+x_{3} \frac{\partial^{2} \varepsilon_{3,0}}{\partial x_{1}^{2}}\right)\right) \\
\varepsilon_{23}= & \varepsilon_{23,0}+x_{1}\left(\frac{\partial \varepsilon_{23,0}}{\partial x_{1}}\right)+\frac{1}{2}\left(x_{1}\right)^{2} \frac{\partial^{2} \varepsilon_{23,0}}{\partial x_{1}^{2}}
\end{aligned}
$$


where, $\kappa_{2,0}$ and $\kappa_{3,0}$ are the curvatures along $x_{2}$ and $x_{3}$ and $\kappa_{1,0}$ is the torsional strain. The strain components shown in Eq. (2) are evaluated at the center of each of the bar members in a truss element to calculate the strain energy of the bar members and subsequently the fundamental truss element. The detailed derivations are presented in Appendix A.

\subsection{Stiffness of an inflatable boom with embedded MFCs}

Figures 3 and 4 depict the carbon fiber inflatable rigidized boom and schematic of the boom and the integrated MFC actuators. This boom is considered the constituent element of the truss structure of Fig. 1. The material and geometrical properties of the inflatable rigidized boom and the MFC are presented in Table 1.
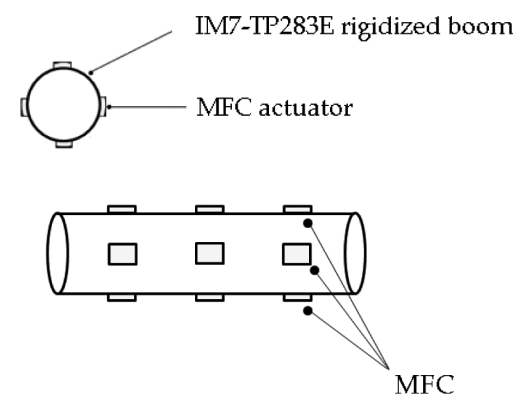

Fig. 3. Schematic of an inflatable boom and attached MFC.

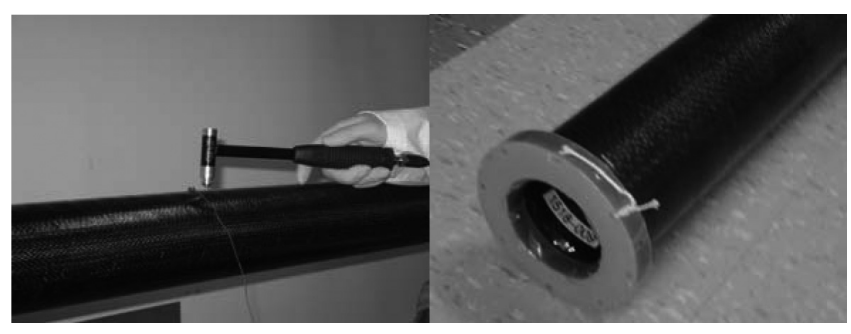

Fig. 4. Carbon fiber inflatable rigidized boom and the end mass made by ILC Dover.

The calculations in following section present the overall stiffness of a boom member with the attached MFCs for a longeron member shown in Fig. 3. The same derivations will be valid for battens and diagonal members. Figure 5 shows schematic of a segment of the carbon fiber longeron boom member and the attached MFCs.

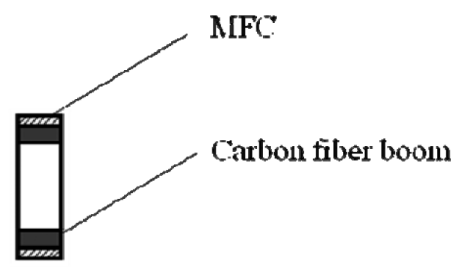

Fig. 5. Carbon fiber boom segment attached to MFCs.

The longitudinal stiffness of this segment is the summation of the boom segment stiffness and the MFC stiffness. Therefore, the overall stiffness for the actuated part (with MFC) can be found as:

$$
K_{L}^{*}=\frac{n_{L} E_{M F C} A_{M F C}}{L_{M F C}}+\frac{E_{L} A_{L}}{L_{M F C}}
$$


where $n_{L}$ is the number of MFCs at the cross section as shown in the Fig. 3. The total stiffness of the longeron is:

$$
\frac{1}{K_{L}}=\frac{1}{K_{P}}+\frac{m_{L}}{K_{L}^{*}}
$$

where $m_{L}$ is the number of MFC actuators along the length of the boom and the stiffness of the non-actuated length (passive length) is given as:

$$
K_{P}=\frac{E_{L} A_{L}}{L_{P}}
$$

Here, $L_{P}$ denotes the passive length of the longeron and can be found as

$$
L_{P}=L_{L}-m_{L} L_{M F C}
$$

The total stiffness of the longeron is then:

$$
K_{L}=\frac{1}{\frac{L_{P}}{E_{L} A_{L}}+\frac{m_{L}}{K_{L}^{*}}}=\frac{E_{L} A_{L} K_{L}^{*}}{\left(L_{L}-m_{L} L_{M F C}\right) K_{L}^{*}+m_{L} E_{L} A_{L}}
$$

Similar derivations are used to find the stiffness values for diagonal and battens. For example for the diagonal members we find:

$$
K_{d}=\frac{E_{d} A_{d} K_{d}^{*}}{\left(L_{d}-m_{d} L_{M F C}\right) K_{d}^{*}+m_{d} E_{d} A_{d}}
$$

where,

$$
K_{d}^{*}=\frac{n_{d} \cdot E_{M F C} \cdot A_{M F C}+E_{d} A_{d}}{L_{M F C}}
$$

Here, $m_{d}$ and $n_{d}$ are the number of MFCs along the length of the diagonal and at the cross section respectively.

Table 1

Material and geometrical properties of the inflatable truss element and MFC

\begin{tabular}{ll}
\hline Property & Value \\
\hline Length of longerons and battens & $3 \mathrm{~m}$ \\
Diameter of longerons, battens and diagonals & $0.0979 \mathrm{~m}$ \\
Elastic modulus of longerons, battens and diagonals & $6.408 \times 10^{10} \mathrm{~N} / \mathrm{m}^{2}$ \\
Density of longerons, battens and diagonals & $1.4293 \times 10^{3} \mathrm{Kg} / \mathrm{m}^{3}$ \\
Length of the MFC actuator & $0.085 \mathrm{~m}$ \\
Width of the MFC actuator & $0.028 \mathrm{~m}$ \\
Thickness of the MFC actuator & $0.3 \mathrm{~mm}$ \\
Elastic modulus of the MFC actuator & $3.0336 \times 10^{10} \mathrm{~N} / \mathrm{m}^{2}$ \\
Tip mass of the longeron, diagonal and batten booms & $0.220 \mathrm{Kg}$ \\
\hline
\end{tabular}

\subsection{Equations of motion}

The strain energy of the fundamental truss element is found using the stiffness values for the inflatable rigidized diagonals, battens and longerons. The detailed derivations are presented in the Appendix. The strain energy of the fundamental element of Fig. 2 is:

$$
\begin{aligned}
U_{e}= & 3 L_{L}^{2} K_{L}\left(\frac{\partial u_{1,0}}{\partial x_{1}}\right)^{2}+\frac{6 L_{L}^{2} L_{b}^{2} K_{L} K_{d}}{K_{d} L_{L}^{2}+4 K_{L} L_{d}^{2}}\left(\frac{\partial u_{2,0}}{\partial x_{1}}-\phi_{3}\right)^{2}+\frac{6 L_{L}^{2} L_{b}^{2} K_{L} K_{d}}{K_{d} L_{L}^{2}+4 K_{L} L_{d}^{2}}\left(\frac{\partial u_{3,0}}{\partial x_{1}}+\phi_{2}\right)^{2} \\
& +\frac{1}{2} L_{L}^{2} L_{b}^{2} K_{L}\left(\frac{\partial \phi_{3}}{\partial x_{1}}\right)^{2}+\frac{1}{2} L_{L}^{2} K_{L} L_{b}^{2}\left(\frac{\partial \phi_{2}}{\partial x_{1}}\right)^{2}+\frac{L_{L}^{2} L_{b}^{4} K_{L} K_{d}}{4\left(K_{d} L_{L}^{2}+K_{L} L_{d}^{2}\right)}\left(\frac{\partial \phi_{1}}{\partial x_{1}}\right)^{2}
\end{aligned}
$$

The kinetic energy of this element is: 


$$
\begin{aligned}
T_{e}= & 3\left(\rho_{b} A_{b} L_{b}+\rho_{L} A_{L} L_{L}+\rho_{d} A_{d} L_{d}\right)\left(\left(\frac{\partial u_{1,0}}{\partial t}\right)^{2}+\left(\frac{\partial u_{2,0}}{\partial t}\right)^{2}+\left(\frac{\partial u_{3,0}}{\partial t}\right)^{2}\right) \\
& +\frac{L_{b}^{2}\left(\rho_{b} A_{b} L_{b}+2 \rho_{L} A_{L} L_{L}+\rho_{d} A_{d} L_{d}\right)}{4}\left(2\left(\frac{\partial \phi_{1,0}}{\partial t}\right)^{2}+\left(\frac{\partial \phi_{2,0}}{\partial t}\right)^{2}+\left(\frac{\partial \phi_{3,0}}{\partial t}\right)^{2}\right)
\end{aligned}
$$

The structure of our interest has a repeated pattern configuration. Therefore, having the kinetic and strain energy expressions for the fundamental truss element, the partial differential equations (PDE) of motion can be found through application of the Hamilton's principle. The displacement components in the kinetic and strain energy expressions are the same as those in a one-dimensional beam specimen and are found as $u_{1,0}, u_{2,0}, u_{3,0}, \phi_{1,0}, \phi_{2,0}$ and $\phi_{3,0}$. The assumptions employed to solve for the strain components of the 3-dimensioanl truss in terms of those for a continuum model are presented in detail in the Appendix. The PDEs are expressed in terms of the stated displacement components (a total of six displacement components). Free-Free boundary conditions are considered for the solution which agrees with conditions of space orbit. The PDEs and their boundary conditions are:

$$
\begin{aligned}
& \left\{\begin{array}{l}
4\left(m_{1}+6 m_{t i p}\right) \frac{\partial^{2} u_{2}}{\partial t^{2}}-C_{2} \frac{\partial}{\partial x}\left(\frac{\partial u_{2}}{\partial x}-\phi_{3}\right)=0 \\
4\left(m_{2}+L_{b}^{2} m_{t i p}\right) \frac{\partial^{2} \phi_{3}}{\partial t^{2}}-4 C_{4} \frac{\partial^{2} \phi_{3}}{\partial x^{2}}-C_{2}\left(\frac{\partial u_{2}}{\partial x}-\phi_{3}\right)=0
\end{array}\right. \\
& \left.\left(\frac{\partial u_{2}}{\partial x}-\phi_{3}\right)\right|_{x=0}=\left.\left(\frac{\partial u_{2}}{\partial x}-\phi_{3}\right)\right|_{x=L_{t}}=\left.\frac{\partial \phi_{3}}{\partial x}\right|_{x=0}=\left.\frac{\partial \phi_{3}}{\partial x}\right|_{x=L_{t}}=0 \\
& \left\{\begin{array}{l}
4\left(m_{1}+6 m_{t i p}\right) \frac{\partial^{2} u_{3}}{\partial t^{2}}-C_{3} \frac{\partial \phi_{2}}{\partial x}\left(\frac{\partial u_{3}}{\partial x}+\phi_{2}\right)=0 \\
4\left(m_{2}+L_{b}^{2} m_{t i p}\right) \frac{\partial^{2} \phi_{2}}{\partial t^{2}}-4 C_{5} \frac{\partial^{2} \phi_{2}}{\partial x^{2}}+C_{3}\left(\frac{\partial u_{3}}{\partial x}+\phi_{2}\right)=0
\end{array}\right. \\
& \left.\left(\frac{\partial u_{3}}{\partial x}+\phi_{2}\right)\right|_{x=0}=\left.\left(\frac{\partial u_{3}}{\partial x}+\phi_{2}\right)\right|_{x=L_{t}}=\left.\frac{\partial \phi_{2}}{\partial x}\right|_{x=0}=\left.\frac{\partial \phi_{2}}{\partial x}\right|_{x=L_{t}}=0 \\
& 2\left(m_{2}+L_{b}^{2} m_{t i p}\right) \frac{\partial^{2} \phi_{1}}{\partial t^{2}}-C_{6} \frac{\partial^{2} \phi_{1}}{\partial x^{2}}=0 \\
& \left.\frac{\partial \phi_{1}}{\partial x}\right|_{x=0}=\left.\frac{\partial \phi_{1}}{\partial x}\right|_{x=L_{t}}=0 \\
& \left(m_{1}+6 m_{t i p}\right) \frac{\partial^{2} u_{1}}{\partial t^{2}}-C_{1} \frac{\partial^{2} u_{1}}{\partial x^{2}}=0 \\
& \left.\frac{\partial u_{1}}{\partial x}\right|_{x=0}=\left.\frac{\partial u_{1}}{\partial x}\right|_{x=L_{t}}=0
\end{aligned}
$$

As shown, each of the bending and the cross section rotation component coordinates are coupled in the form of two equations. This is similar to the bending equations of motion for a Timoshenko beam. Also, the torsional and longitudinal equations are similar to those of a bar member. Solution of these PDEs results in the natural frequencies of the system for each of the vibration coordinates. The solution procedure for these PDEs is very similar to [23] where the solution is expanded in terms of mode shape parameters (spatial terms) and frequencies (temporal solution). The boundary conditions are applied to the expanded solution to relate the mode shape parameters to the frequencies. Subsequently the frequencies are found from the roots of the determinant of the resultant matrix from the boundary conditions. The numerical results are presented in the following sections. Ref. [23] presents the detailed solution for PDEs of this form and the natural frequencies. 
Table 2

Number of MFCs along the length of members for various configurations

\begin{tabular}{lc}
\hline Property & Value \\
\hline longeron (configuration 2) & 5 \\
diagonal (configuration 2) & 7 \\
longeron (configuration 3) & 10 \\
diagonal (configuration 3) & 14 \\
\hline
\end{tabular}

Table 3

Bending natural frequencies of a radar truss for $t_{L}=t_{b}=t_{d}=4.8 \times 10^{-4} \mathrm{~m}$ and $L_{\text {tot }}=120 \mathrm{~m}$ in $(\mathrm{rad} / \mathrm{sec})$

\begin{tabular}{ccc}
\multicolumn{1}{c}{$L_{\text {tot }}=120 \mathrm{~m}$ in $(\mathrm{rad} / \mathrm{sec})$} \\
\hline Configuration 1 & Configuration 2 & PFC $^{1}$ \\
\hline 6.258 & 6.301 & 0.687 \\
37.049 & 17.167 & 0.692 \\
53.029 & 33.063 & 0.697 \\
77.041 & 53.398 & 0.696 \\
104.260 & 77.577 & 0.696 \\
134.110 & 104.985 & 0.695 \\
166.073 & 135.042 & 0.695 \\
199.703 & 167.226 & 0.694 \\
234.626 & 201.089 & 0.694 \\
270.536 & 236.254 & 0.694 \\
307.186 & 272.411 & 0.693 \\
344.380 & 309.315 & 0.693 \\
381.962 & 346.766 & 0.693 \\
419.812 & 384.608 & 0.693 \\
457.836 & 422.719 & 0.692 \\
495.964 & 461.006 & 0.692 \\
534.139 & 499.397 & 0.692 \\
572.320 & 537.836 & 0.692 \\
610.480 & 576.281 & 0.692 \\
\hline PFC: Percentage Frequency Change. &
\end{tabular}

Table 4

Torsional natural frequencies of a radar truss for $t_{L}=t_{b}=t_{d}=4.8 \times 10^{-4} \mathrm{~m}$ and $\mathrm{L}_{\text {tot }}=120 \mathrm{~m}$ in $(\mathrm{rad} / \mathrm{sec})$

\begin{tabular}{ccc}
\hline Configuration 1 & Configuration 2 & PFC \\
\hline 26.294 & 26.476 & 0.692 \\
52.589 & 52.952 & 0.690 \\
78.884 & 79.429 & 0.691 \\
105.178 & 105.905 & 0.691 \\
131.473 & 132.381 & 0.691 \\
157.767 & 158.857 & 0.691 \\
184.062 & 185.334 & 0.691 \\
210.356 & 211.810 & 0.691 \\
236.651 & 238.286 & 0.691 \\
262.945 & 264.762 & 0.691 \\
289.240 & 291.238 & 0.691 \\
315.534 & 317.715 & 0.691 \\
341.829 & 344.191 & 0.691 \\
368.123 & 370.667 & 0.691 \\
394.418 & 397.143 & 0.691 \\
420.713 & 423.619 & 0.691 \\
447.007 & 450.096 & 0.691 \\
473.302 & 476.572 & 0.691 \\
499.596 & 503.048 & 0.691 \\
525.891 & 529.524 & 0.691 \\
\hline
\end{tabular}

\section{Numerical results}

The geometry and material properties of the truss, inflatable boom and the MFCs are given in Table 1. Different configurations for MFCs are considered in this work for the purpose of comparison. The first configuration considers a structure made of the booms shown in Fig. 4 with no MFCs attached. The second and third configurations have 4 MFCs along the circumference as shown in Fig. 3 for each of the diagonal and the longeron members. The number of MFCs along the length of each of these members is shown in Table 2. MFCs are equally spaced on all these members. The result for the frequencies of lattice structures with various lengths and thickness values are presented in this section. As shown in Eqs (12) and (13), the coordinates for bending and rotation of the cross section are coupled. Therefore, the frequencies for these two coordinates are the same. Also, the results are identical for bending along $x_{2}$ and $x_{3}$ axes due to the symmetric diagonal pattern for this structure. Figures 6 and 7 show the bending frequencies of a lattice structure made of the boom member shown in Fig. 4. The results are presented for various truss lengths and boom thickness values.

A Finite Element Analysis (FEA) code was developed in MATLAB using the method presented in [24] to validate the accuracy of the natural frequencies of the developed model. The FEA code consists of 903 linear elements and 810 degrees of freedom. The comparison with the FEA results is presented in Figs 6, 7 and 8; the results are shown to be in excellent agreement. As expected and also clear from these figures, the frequencies decrease as the length of the truss increases. The same bar lengths were used in the construction of the elements for all truss structures. Also, the results show higher accuracy for longer lengths. This is also expected as the overall length to the element size ratio is directly related to the accuracy of frequency estimations. Another noteworthy point about these figures is that the FEA overestimates the frequencies. This is because FEA solvers find the higher bound of a Rayleigh Quotient in 


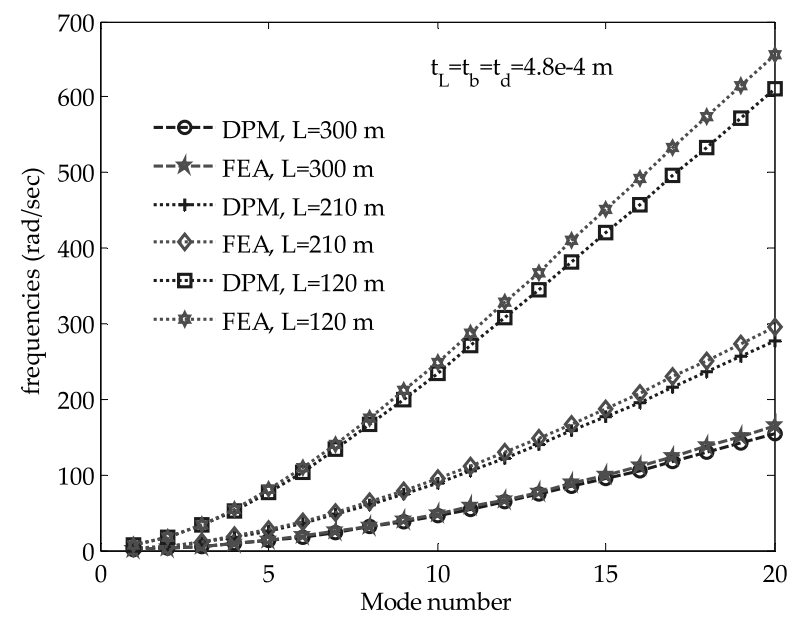

Fig. 6. Frequency comparison of DPM and FEA for satellite truss of various lengths (no actuators- configuration 1).
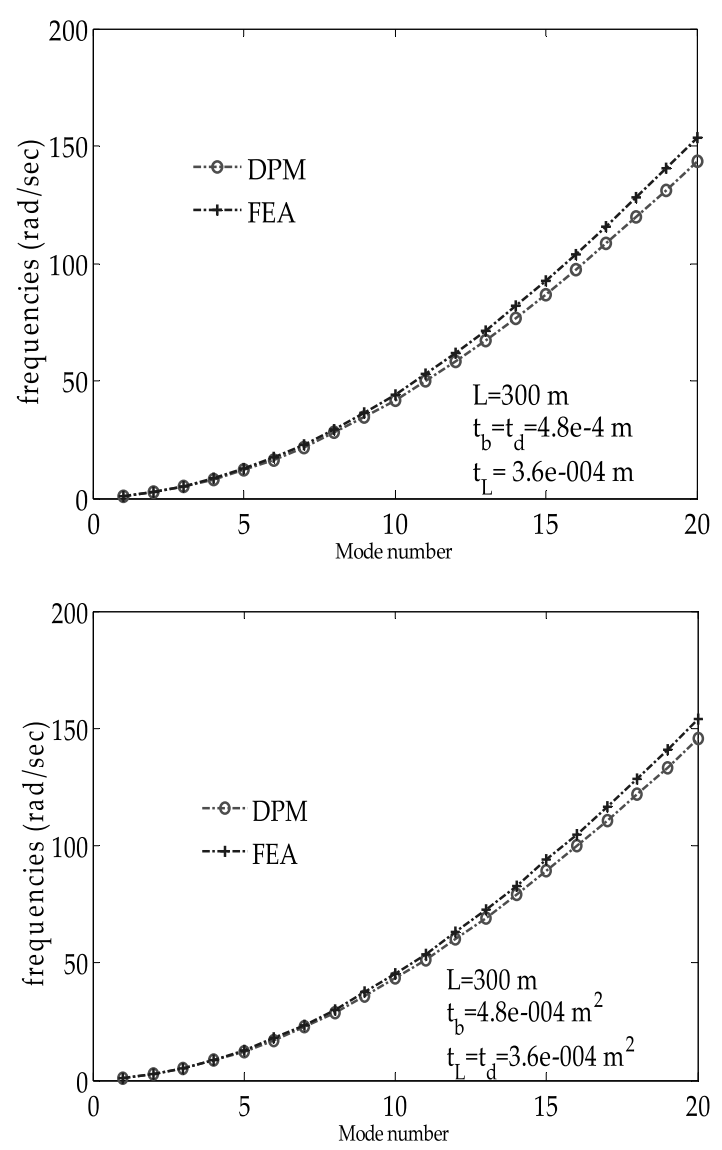

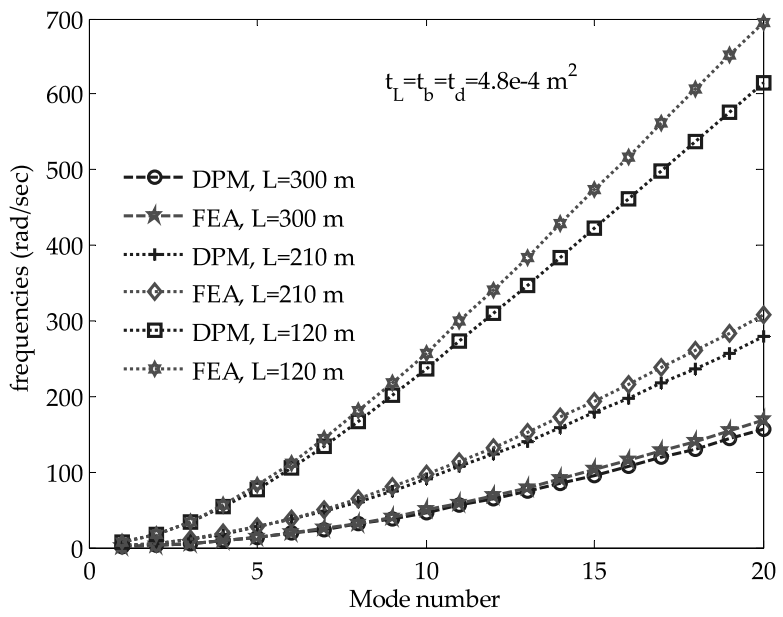

Fig. 7. Frequency comparison of DPM and FEA for satellite truss of various lengths (actuator configuration 2).
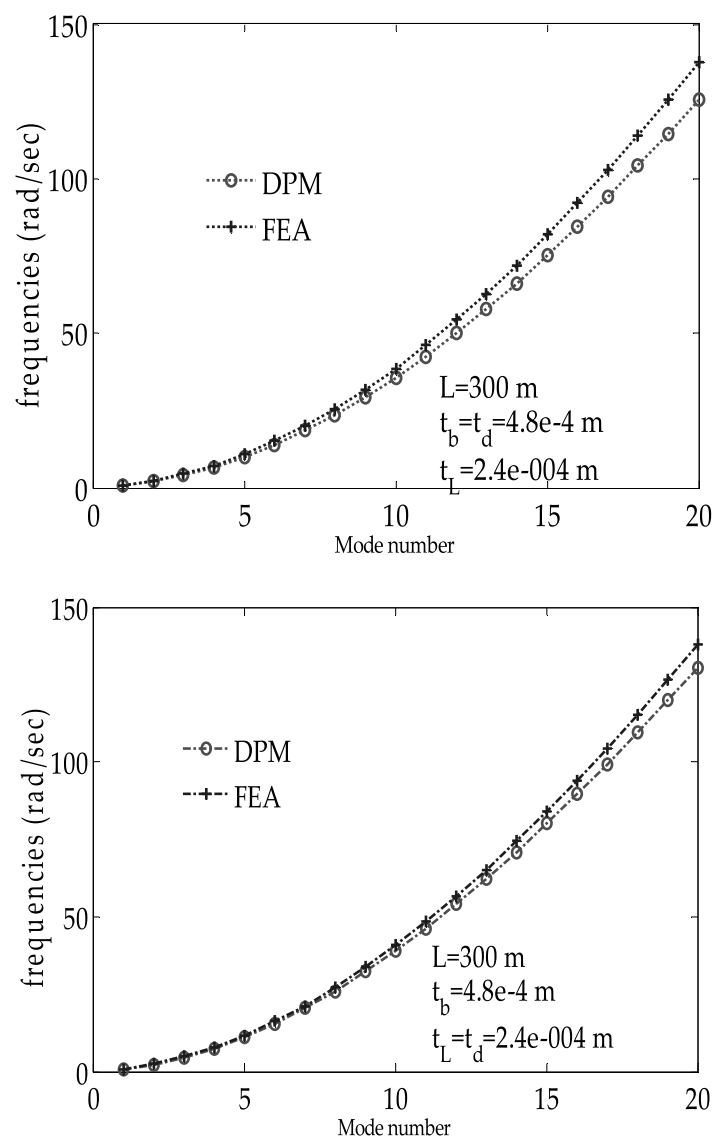

Fig. 8. Frequency comparison of DPM and FEA for satellite truss of various longeron thickness (no actuators- configuration 1). 
Table 5

Longitudinal natural frequencies of a radar truss for $t_{L}=t_{b}=t_{d}=4.8 \times 10^{-4} \mathrm{~m}$ and

\begin{tabular}{ccc}
$L_{t o t}=120 \mathrm{~m}$ in $(\mathrm{rad} / \mathrm{sec})$ & \\
\hline Configuration 1 & Configuration 2 & PFC \\
\hline 86.474 & 87.076 & 0.696 \\
172.948 & 174.152 & 0.696 \\
259.422 & 261.228 & 0.696 \\
345.895 & 348.303 & 0.696 \\
432.369 & 435.379 & 0.696 \\
518.843 & 522.455 & 0.696 \\
605.317 & 609.531 & 0.696 \\
691.791 & 696.607 & 0.696 \\
778.265 & 783.683 & 0.696 \\
864.738 & 870.758 & 0.696 \\
951.212 & 957.834 & 0.696 \\
1037.690 & 1044.910 & 0.696 \\
1124.160 & 1131.990 & 0.696 \\
1210.630 & 1219.060 & 0.696 \\
1297.110 & 1306.140 & 0.696 \\
1383.580 & 1393.210 & 0.696 \\
1470.060 & 1480.290 & 0.696 \\
1556.530 & 1567.370 & 0.696 \\
1643.000 & 1654.440 & 0.696 \\
1729.480 & 1741.520 & 0.696 \\
\hline & &
\end{tabular}

Table 7

Torsional natural frequencies of a radar truss for $t_{L}=t_{b}=t_{d}=4.8 \times 10^{-4} \mathrm{~m}$ and $L_{\text {tot }}=210 \mathrm{~m}$ in $(\mathrm{rad} / \mathrm{sec})$

\begin{tabular}{ccc}
\hline Configuration 1 & Configuration 2 & PFC \\
\hline 15.025 & 15.129 & 0.692 \\
30.051 & 30.258 & 0.689 \\
45.076 & 45.388 & 0.692 \\
60.102 & 60.517 & 0.690 \\
75.127 & 75.646 & 0.691 \\
90.153 & 90.776 & 0.691 \\
105.178 & 105.905 & 0.691 \\
120.204 & 121.034 & 0.691 \\
135.229 & 136.163 & 0.691 \\
150.254 & 151.293 & 0.692 \\
165.28 & 166.422 & 0.691 \\
180.305 & 181.551 & 0.691 \\
195.331 & 196.680 & 0.691 \\
210.356 & 211.810 & 0.691 \\
225.382 & 226.939 & 0.691 \\
240.407 & 242.068 & 0.691 \\
255.433 & 257.198 & 0.691 \\
270.458 & 272.327 & 0.691 \\
285.484 & 287.456 & 0.691 \\
300.509 & 302.585 & 0.691 \\
\hline & &
\end{tabular}

Table 6

Bending natural frequencies of a radar truss for $t_{L}=t_{b}=t_{d}=4.8 \times 10^{-4} \mathrm{~m}$ and $L_{\text {tot }}=210 \mathrm{~m}$ in $(\mathrm{rad} / \mathrm{sec})$

\begin{tabular}{rrr}
\hline Configuration 1 & Configuration 2 & PFC \\
\hline 2.049 & 2.064 & 0.727 \\
5.627 & 5.666 & 0.688 \\
10.965 & 11.042 & 0.697 \\
17.970 & 18.104 & 0.740 \\
26.586 & 26.771 & 0.691 \\
36.688 & 36.944 & 0.693 \\
48.181 & 48.516 & 0.690 \\
60.950 & 61.374 & 0.691 \\
74.882 & 75.403 & 0.691 \\
89.864 & 90.488 & 0.690 \\
105.785 & 106.520 & 0.690 \\
122.542 & 123.393 & 0.690 \\
140.038 & 141.010 & 0.689 \\
158.183 & 159.281 & 0.689 \\
176.895 & 178.123 & 0.689 \\
196.100 & 197.460 & 0.689 \\
215.730 & 217.227 & 0.689 \\
235.727 & 237.362 & 0.689 \\
256.037 & 257.813 & 0.689 \\
276.615 & 278.533 & 0.689 \\
\hline & &
\end{tabular}

Table 8

Longitudinal natural frequencies of a radar truss for $t_{L}=t_{b}=t_{d}=4.8 \times 10^{-4} \mathrm{~m}$ and $L_{\text {tot }}=210 \mathrm{~m}$ in $(\mathrm{rad} / \mathrm{sec})$

\begin{tabular}{ccc}
\multicolumn{1}{l}{$L_{t o t}=210 \mathrm{~m}$ in $(\mathrm{rad} / \mathrm{sec})$} \\
\hline Configuration 1 & Configuration 2 & PFC \\
\hline 49.414 & 49.758 & 0.696 \\
98.827 & 99.515 & 0.696 \\
148.240 & 149.273 & 0.697 \\
197.654 & 199.030 & 0.696 \\
247.068 & 248.788 & 0.696 \\
296.482 & 298.546 & 0.696 \\
345.895 & 348.303 & 0.696 \\
395.309 & 398.061 & 0.696 \\
444.723 & 447.819 & 0.696 \\
494.136 & 497.576 & 0.696 \\
543.550 & 547.334 & 0.696 \\
592.963 & 597.091 & 0.696 \\
642.377 & 646.849 & 0.696 \\
691.791 & 696.607 & 0.696 \\
741.204 & 746.364 & 0.696 \\
790.618 & 796.122 & 0.696 \\
840.032 & 845.880 & 0.696 \\
889.445 & 895.637 & 0.696 \\
938.859 & 945.395 & 0.696 \\
988.272 & 995.152 & 0.696 \\
\hline
\end{tabular}


Table 9

Bending natural frequencies of a radar truss for $t_{L}=t_{b}=t_{d}=$ $4.8 \times 10^{-4} \mathrm{~m}$ and $L_{t o t}=300 \mathrm{~m}$ in $(\mathrm{rad} / \mathrm{sec})$

\begin{tabular}{ccccc}
\hline Configuration 1 & Configuration 2 & PFC & Configuration 3 & PFC \\
\hline 1.005 & 1.012 & 0.696 & 1.019 & 1.393 \\
2.765 & 2.784 & 0.687 & 2.804 & 1.411 \\
5.404 & 5.442 & 0.703 & 5.480 & 1.406 \\
8.897 & 8.959 & 0.697 & 9.022 & 1.405 \\
13.222 & 13.314 & 0.696 & 13.408 & 1.407 \\
18.354 & 18.482 & 0.697 & 18.612 & 1.406 \\
24.263 & 24.432 & 0.696 & 24.604 & 1.405 \\
30.915 & 31.131 & 0.699 & 31.350 & 1.407 \\
38.275 & 38.542 & 0.698 & 38.814 & 1.408 \\
46.306 & 46.628 & 0.695 & 46.957 & 1.406 \\
54.968 & 55.350 & 0.695 & 55.741 & 1.406 \\
64.222 & 64.669 & 0.696 & 65.125 & 1.406 \\
74.030 & 74.545 & 0.696 & 75.070 & 1.405 \\
84.353 & 84.939 & 0.695 & 85.538 & 1.405 \\
95.152 & 95.813 & 0.695 & 96.489 & 1.405 \\
106.392 & 107.131 & 0.695 & 107.886 & 1.404 \\
118.037 & 118.857 & 0.695 & 119.695 & 1.405 \\
130.054 & 130.958 & 0.695 & 131.880 & 1.404 \\
142.410 & 143.400 & 0.695 & 144.411 & 1.405 \\
155.079 & 156.156 & 0.694 & 157.256 & 1.404 \\
\hline & & & &
\end{tabular}

Table 11

Longitudinal natural frequencies of a radar truss for $t_{L}=t_{b}=t_{d}=$ $4.8 \times 10^{-4} \mathrm{~m}$ and $L_{t o t}=300 \mathrm{~m}$ in $(\mathrm{rad} / \mathrm{sec})$

\begin{tabular}{ccccc}
$4.8 \times 10$ & $m$ and $L_{\text {tot }}=300 \mathrm{~m}$ in $(\mathrm{rad} / \mathrm{sec}$ ) \\
\hline Configuration 1 & Configuration 2 & PFC & Configuration 3 & PFC \\
\hline 34.589 & 34.830 & 0.697 & 35.076 & 1.408 \\
69.179 & 69.661 & 0.697 & 70.152 & 1.406 \\
103.770 & 104.491 & 0.695 & 105.229 & 1.406 \\
138.358 & 139.321 & 0.696 & 140.305 & 1.407 \\
172.948 & 174.152 & 0.696 & 175.381 & 1.407 \\
207.537 & 208.982 & 0.696 & 210.457 & 1.407 \\
242.127 & 243.812 & 0.696 & 245.534 & 1.407 \\
276.716 & 278.643 & 0.696 & 280.610 & 1.407 \\
311.306 & 313.473 & 0.696 & 315.686 & 1.407 \\
345.895 & 348.303 & 0.696 & 350.762 & 1.407 \\
380.485 & 383.134 & 0.696 & 385.839 & 1.407 \\
415.074 & 417.964 & 0.696 & 420.915 & 1.407 \\
449.664 & 452.794 & 0.696 & 455.991 & 1.407 \\
484.253 & 487.625 & 0.696 & 491.067 & 1.407 \\
518.843 & 522.455 & 0.696 & 526.144 & 1.407 \\
553.433 & 557.285 & 0.696 & 561.220 & 1.407 \\
588.022 & 592.116 & 0.696 & 596.296 & 1.407 \\
622.612 & 626.946 & 0.696 & 631.372 & 1.407 \\
657.201 & 661.776 & 0.696 & 666.449 & 1.407 \\
691.791 & 696.607 & 0.696 & 701.525 & 1.407 \\
\hline
\end{tabular}

Table 10

Torsional natural frequencies of a radar truss for $t_{L}=t_{b}=t_{d}=$ $4.8 \times 10^{-4} \mathrm{~m}$ and $L_{t o t}=300 \mathrm{~m}$ in $(\mathrm{rad} / \mathrm{sec})$

\begin{tabular}{ccccc}
\hline Configuration 1 & Configuration 2 & PFC & Configuration 3 & PFC \\
\hline 10.518 & 10.590 & 0.684 & 10.665 & 1.398 \\
21.036 & 21.181 & 0.689 & 21.329 & 1.393 \\
31.553 & 31.772 & 0.694 & 31.994 & 1.398 \\
42.071 & 42.362 & 0.692 & 42.659 & 1.398 \\
52.589 & 52.952 & 0.690 & 53.323 & 1.396 \\
63.107 & 63.543 & 0.691 & 63.988 & 1.396 \\
73.625 & 74.133 & 0.690 & 74.653 & 1.396 \\
84.142 & 84.724 & 0.692 & 85.318 & 1.398 \\
94.660 & 95.314 & 0.691 & 95.982 & 1.397 \\
105.178 & 105.905 & 0.691 & 106.647 & 1.397 \\
115.696 & 116.495 & 0.691 & 117.312 & 1.397 \\
126.214 & 127.086 & 0.691 & 127.976 & 1.396 \\
136.732 & 137.676 & 0.690 & 138.641 & 1.396 \\
147.249 & 148.267 & 0.691 & 149.306 & 1.397 \\
157.767 & 158.857 & 0.691 & 159.970 & 1.396 \\
168.285 & 169.448 & 0.691 & 170.635 & 1.396 \\
178.803 & 180.038 & 0.691 & 181.300 & 1.396 \\
189.321 & 190.629 & 0.691 & 191.964 & 1.396 \\
199.838 & 201.219 & 0.691 & 202.629 & 1.397 \\
210.356 & 211.810 & 0.691 & 213.294 & 1.397 \\
\hline & & & &
\end{tabular}

Table 12

Bending natural frequencies of a truss for $t_{b}=t_{d}=4.8 \times$ $10^{-4} \mathrm{~m} t_{L}=3.6 \times 10^{-4} \mathrm{~m}$ and $L_{\text {tot }}=300 \mathrm{~m}$ in $(\mathrm{rad} / \mathrm{sec})$

\begin{tabular}{ccccc}
$10^{-4} m t_{L}=3.6 \times 10^{-4} m$ and $L_{t o t}=300 m$ in $(\mathrm{rad} / \mathrm{sec})$ \\
\hline Configuration 1 & Configuration 2 & PFC & Configuration 3 & PFC \\
\hline 0.898 & 0.906 & 0.891 & 0.914 & 1.782 \\
2.470 & 2.494 & 0.972 & 2.517 & 1.903 \\
4.835 & 4.878 & 0.889 & 4.923 & 1.820 \\
7.966 & 8.038 & 0.904 & 8.112 & 1.833 \\
11.852 & 11.959 & 0.903 & 12.068 & 1.823 \\
16.473 & 16.620 & 0.892 & 16.772 & 1.815 \\
21.807 & 22.002 & 0.894 & 22.202 & 1.811 \\
27.830 & 28.100 & 0.970 & 28.334 & 1.811 \\
34.516 & 34.823 & 0.889 & 35.138 & 1.802 \\
41.835 & 42.207 & 0.889 & 42.588 & 1.800 \\
49.759 & 50.200 & 0.886 & 50.652 & 1.795 \\
58.258 & 58.772 & 0.882 & 59.300 & 1.789 \\
67.300 & 67.892 & 0.880 & 68.500 & 1.783 \\
76.854 & 77.528 & 0.877 & 78.220 & 1.777 \\
86.891 & 87.650 & 0.873 & 88.430 & 1.771 \\
97.379 & 98.227 & 0.871 & 99.098 & 1.765 \\
108.290 & 109.230 & 0.868 & 110.195 & 1.759 \\
119.595 & 120.629 & 0.865 & 121.690 & 1.752 \\
131.266 & 132.396 & 0.861 & 133.557 & 1.745 \\
143.276 & 144.506 & 0.859 & 145.768 & 1.739 \\
\hline & & & &
\end{tabular}


Table 13

Torsional natural frequencies of a radar truss for $t_{b}=t_{d}=4.8 \times$ $10^{-4} \mathrm{~m}_{L}=3.6 \times 10^{-4} \mathrm{~m}$ and $L_{\text {tot }}=300 \mathrm{~m}$ in $(\mathrm{rad} / \mathrm{sec})$

\begin{tabular}{ccccc}
\hline Configuration 1 & Configuration 2 & PFC & Configuration 3 & PFC \\
\hline 10.553 & 10.633 & 0.758 & 10.714 & 1.526 \\
21.105 & 21.265 & 0.758 & 21.428 & 1.530 \\
31.658 & 31.897 & 0.755 & 32.143 & 1.532 \\
42.210 & 42.530 & 0.758 & 42.857 & 1.533 \\
52.763 & 53.162 & 0.756 & 53.571 & 1.531 \\
63.315 & 63.795 & 0.758 & 64.285 & 1.532 \\
73.868 & 74.427 & 0.757 & 74.999 & 1.531 \\
84.420 & 85.060 & 0.758 & 85.714 & 1.533 \\
94.973 & 95.692 & 0.757 & 96.428 & 1.532 \\
105.526 & 106.325 & 0.757 & 107.142 & 1.531 \\
116.078 & 116.957 & 0.757 & 117.856 & 1.532 \\
126.631 & 127.589 & 0.756 & 128.570 & 1.531 \\
137.183 & 138.222 & 0.757 & 139.284 & 1.532 \\
147.736 & 148.854 & 0.757 & 149.999 & 1.532 \\
158.288 & 159.487 & 0.757 & 160.713 & 1.532 \\
168.841 & 170.119 & 0.757 & 171.427 & 1.532 \\
179.393 & 180.752 & 0.758 & 182.141 & 1.532 \\
189.946 & 191.384 & 0.757 & 192.855 & 1.531 \\
200.499 & 202.017 & 0.757 & 203.570 & 1.532 \\
211.051 & 212.649 & 0.757 & 214.284 & 1.532 \\
\hline & & & &
\end{tabular}

Table 15

Bending natural frequencies of a radar truss for $t_{b}=4.8 \times$ $10^{-4} \mathrm{~m} t_{L}=t_{d}=3.6 \times 10^{-4} \mathrm{~m}$ and $L_{t o t}=300 \mathrm{~m}$ in $(\mathrm{rad} / \mathrm{sec})$

\begin{tabular}{ccccc}
\hline Configuration 1 & Configuration 2 & PFC & Configuration 3 & PFC \\
\hline 0.942 & 0.951 & 0.955 & 0.959 & 1.805 \\
2.592 & 2.616 & 0.926 & 2.639 & 1.813 \\
5.067 & 5.112 & 0.888 & 5.159 & 1.816 \\
8.342 & 8.417 & 0.899 & 8.494 & 1.822 \\
12.397 & 12.509 & 0.903 & 12.624 & 1.831 \\
17.209 & 17.364 & 0.901 & 17.524 & 1.830 \\
22.749 & 22.954 & 0.901 & 23.165 & 1.829 \\
28.987 & 29.248 & 0.900 & 29.516 & 1.825 \\
35.888 & 36.211 & 0.900 & 36.543 & 1.825 \\
43.417 & 43.808 & 0.901 & 44.210 & 1.826 \\
51.539 & 52.003 & 0.900 & 52.480 & 1.826 \\
60.216 & 60.758 & 0.900 & 61.316 & 1.827 \\
69.412 & 70.037 & 0.900 & 70.769 & 1.955 \\
79.090 & 79.803 & 0.902 & 80.534 & 1.826 \\
89.216 & 90.019 & 0.900 & 90.845 & 1.826 \\
99.755 & 100.653 & 0.900 & 101.575 & 1.824 \\
110.674 & 111.670 & 0.900 & 112.693 & 1.824 \\
121.941 & 123.038 & 0.900 & 124.166 & 1.825 \\
133.528 & 134.729 & 0.899 & 135.963 & 1.824 \\
145.405 & 146.713 & 0.900 & 148.057 & 1.824 \\
\hline & & & &
\end{tabular}

Table 14

Longitudinal natural frequencies of a radar truss for $t_{b}=t_{d}=4.8$ $\times 10^{-4} \mathrm{~m} t_{L}=3.6 \times 10^{-4} \mathrm{~m}$ and $L_{t o t}=300 \mathrm{~m}$ in $(\mathrm{rad} / \mathrm{sec})$

\begin{tabular}{ccccc}
\hline Configuration 1 & Configuration 2 & PFC & Configuration 3 & PFC \\
\hline 30.910 & 31.189 & 0.903 & 31.476 & 1.831 \\
61.821 & 62.378 & 0.901 & 62.951 & 1.828 \\
92.731 & 93.568 & 0.903 & 94.427 & 1.829 \\
123.642 & 124.757 & 0.902 & 125.903 & 1.829 \\
154.552 & 155.946 & 0.902 & 157.378 & 1.828 \\
185.463 & 187.135 & 0.902 & 188.854 & 1.828 \\
216.373 & 218.324 & 0.902 & 220.329 & 1.828 \\
247.283 & 249.513 & 0.901 & 251.805 & 1.828 \\
278.194 & 280.703 & 0.901 & 283.281 & 1.828 \\
309.104 & 311.892 & 0.902 & 314.756 & 1.828 \\
340.015 & 343.081 & 0.902 & 346.232 & 1.828 \\
370.925 & 374.270 & 0.901 & 377.708 & 1.828 \\
401.836 & 405.459 & 0.901 & 409.183 & 1.828 \\
432.746 & 436.649 & 0.901 & 440.659 & 1.828 \\
463.656 & 467.838 & 0.902 & 472.134 & 1.828 \\
494.567 & 499.027 & 0.901 & 503.610 & 1.828 \\
525.477 & 530.216 & 0.901 & 535.086 & 1.828 \\
556.388 & 561.405 & 0.901 & 566.561 & 1.828 \\
587.298 & 592.595 & 0.901 & 598.037 & 1.828 \\
618.209 & 623.784 & 0.901 & 629.513 & 1.828 \\
\hline & & & &
\end{tabular}

Table 16

Torsional natural frequencies of a radar truss for $t_{b}=4.8 \times$ $10^{-4} \mathrm{~m} t_{L}=t_{d}=3.6 \times 10^{-4} \mathrm{~m}$ and $L_{t o t}=300 \mathrm{~m}$ in $(\mathrm{rad} / \mathrm{sec})$

\begin{tabular}{crrrr}
\multicolumn{1}{c}{$m t_{L}=t_{d}=3.6 \times 10$} & \multicolumn{3}{c}{ and $L_{t o t}=300 \mathrm{~m}$ in $(\mathrm{rad} / \mathrm{sec})$} \\
\hline Configuration 1 & Configuration 2 & PFC & Configuration 3 & PFC \\
\hline 9.863 & 9.951 & 0.892 & 10.042 & 1.815 \\
19.725 & 19.902 & 0.897 & 20.083 & 1.815 \\
29.588 & 29.853 & 0.895 & 30.125 & 1.814 \\
39.451 & 39.804 & 0.894 & 40.167 & 1.814 \\
49.313 & 49.755 & 0.896 & 50.208 & 1.814 \\
59.176 & 59.706 & 0.895 & 60.245 & 1.806 \\
69.039 & 69.657 & 0.895 & 70.292 & 1.814 \\
78.902 & 79.608 & 0.894 & 80.333 & 1.813 \\
88.764 & 89.559 & 0.895 & 90.375 & 1.814 \\
98.627 & 99.510 & 0.895 & 100.417 & 1.814 \\
108.490 & 109.461 & 0.895 & 110.458 & 1.814 \\
118.352 & 119.412 & 0.895 & 120.500 & 1.814 \\
128.215 & 129.363 & 0.895 & 130.541 & 1.814 \\
138.078 & 139.313 & 0.894 & 140.583 & 1.814 \\
147.940 & 149.264 & 0.895 & 150.625 & 1.814 \\
157.803 & 159.215 & 0.894 & 160.666 & 1.814 \\
167.666 & 169.166 & 0.894 & 170.708 & 1.814 \\
177.528 & 179.117 & 0.895 & 180.750 & 1.814 \\
187.391 & 189.068 & 0.894 & 190.791 & 1.814 \\
197.254 & 199.019 & 0.894 & 200.833 & 1.814 \\
\hline & & & &
\end{tabular}


Table 17

Longitudinal natural frequencies of a radar truss for $t_{b}=4.8 \times$ $10^{-4} \mathrm{~m}_{L}=t_{d}=3.6 \times 10^{-4} \mathrm{~m}$ and $L_{t o t}=300 \mathrm{~m}$ in $(\mathrm{rad} / \mathrm{sec})$

\begin{tabular}{ccccc}
\hline Configuration 1 & Configuration 2 & PFC & Configuration 3 & PFC \\
\hline 32.432 & 32.724 & 0.900 & 33.025 & 1.828 \\
64.863 & 65.448 & 0.901 & 66.049 & 1.828 \\
97.295 & 98.172 & 0.901 & 99.074 & 1.828 \\
129.727 & 130.900 & 0.904 & 132.099 & 1.828 \\
162.158 & 163.621 & 0.902 & 165.123 & 1.828 \\
194.590 & 196.345 & 0.901 & 198.148 & 1.828 \\
227.022 & 229.069 & 0.901 & 231.173 & 1.828 \\
259.453 & 261.793 & 0.901 & 264.197 & 1.828 \\
291.885 & 294.517 & 0.901 & 297.222 & 1.828 \\
324.317 & 327.241 & 0.901 & 330.247 & 1.828 \\
356.748 & 359.965 & 0.901 & 363.271 & 1.828 \\
389.180 & 392.690 & 0.902 & 396.296 & 1.828 \\
421.611 & 425.414 & 0.902 & 429.321 & 1.828 \\
454.043 & 458.138 & 0.902 & 462.345 & 1.828 \\
486.475 & 490.862 & 0.902 & 495.370 & 1.828 \\
518.906 & 523.586 & 0.902 & 528.395 & 1.828 \\
551.338 & 556.310 & 0.902 & 561.419 & 1.828 \\
583.770 & 589.034 & 0.902 & 594.444 & 1.828 \\
616.201 & 621.758 & 0.902 & 627.469 & 1.828 \\
648.633 & 654.483 & 0.902 & 660.493 & 1.828 \\
\hline & & & &
\end{tabular}

Table 19

Torsional natural frequencies of a radar truss for $t_{b}=t_{d}=4.8 \times$ $10^{-4} \mathrm{~m}_{L}=2.4 \times 10^{-4} \mathrm{~m}$ and $L_{t o t}=300 \mathrm{~m}$ in $(\mathrm{rad} / \mathrm{sec})$

\begin{tabular}{ccccc}
\hline Configuration 1 & Configuration 2 & PFC & Configuration 3 & PFC \\
\hline 10.294 & 10.390 & 0.932 & 10.489 & 1.894 \\
20.588 & 20.780 & 0.932 & 20.978 & 1.894 \\
30.883 & 31.171 & 0.932 & 31.467 & 1.891 \\
41.177 & 41.561 & 0.932 & 41.956 & 1.891 \\
51.471 & 51.951 & 0.932 & 52.445 & 1.892 \\
61.765 & 62.341 & 0.932 & 62.934 & 1.892 \\
72.059 & 72.731 & 0.932 & 73.422 & 1.891 \\
82.353 & 83.121 & 0.932 & 83.911 & 1.891 \\
92.648 & 93.512 & 0.932 & 94.400 & 1.891 \\
102.942 & 103.902 & 0.932 & 104.889 & 1.891 \\
113.236 & 114.292 & 0.932 & 115.378 & 1.891 \\
123.530 & 124.682 & 0.932 & 125.867 & 1.891 \\
133.824 & 135.072 & 0.932 & 136.356 & 1.892 \\
144.119 & 145.463 & 0.932 & 146.845 & 1.891 \\
154.413 & 155.853 & 0.932 & 157.334 & 1.891 \\
164.707 & 166.243 & 0.932 & 167.823 & 1.891 \\
175.001 & 176.633 & 0.932 & 178.312 & 1.892 \\
185.295 & 187.023 & 0.932 & 188.801 & 1.892 \\
195.590 & 197.414 & 0.932 & 199.290 & 1.891 \\
205.884 & 207.804 & 0.932 & 209.779 & 1.891 \\
\hline & & & &
\end{tabular}

Table 18

Bending natural frequencies of a radar truss for $t_{b}=t_{d}=4.8 \times$ $10^{-4} \mathrm{~m} t_{L}=2.4 \times 10^{-4} \mathrm{~m}$ and $L_{\text {tot }}=300 \mathrm{~m}$ in $(\mathrm{rad} / \mathrm{sec})$

\begin{tabular}{rrrrr}
\hline Configuration 1 & Configuration 2 & PFC & Configuration 3 & PFC \\
\hline 0.758 & 0.768 & 1.319 & 0.778 & 2.639 \\
2.088 & 2.115 & 1.293 & 2.143 & 2.634 \\
4.087 & 4.139 & 1.272 & 4.193 & 2.594 \\
6.740 & 6.826 & 1.276 & 6.915 & 2.596 \\
10.038 & 10.165 & 1.265 & 10.298 & 2.590 \\
13.969 & 14.146 & 1.267 & 14.330 & 2.584 \\
18.519 & 18.753 & 1.264 & 18.997 & 2.581 \\
23.673 & 23.971 & 1.259 & 24.282 & 2.573 \\
29.412 & 29.782 & 1.258 & 30.166 & 2.564 \\
35.719 & 36.167 & 1.254 & 36.632 & 2.556 \\
42.573 & 43.105 & 1.250 & 43.657 & 2.546 \\
49.954 & 50.575 & 1.243 & 51.220 & 2.534 \\
57.841 & 58.556 & 1.236 & 59.300 & 2.522 \\
66.211 & 67.026 & 1.231 & 67.872 & 2.509 \\
75.042 & 75.962 & 1.226 & 76.916 & 2.497 \\
84.313 & 85.341 & 1.219 & 86.407 & 2.484 \\
94.001 & 95.142 & 1.214 & 96.324 & 2.471 \\
104.086 & 105.342 & 1.207 & 106.644 & 2.458 \\
114.545 & 115.920 & 1.200 & 117.344 & 2.444 \\
125.358 & 126.854 & 1.193 & 128.405 & 2.431 \\
\hline & & & &
\end{tabular}

Table 20

Longitudinal natural frequencies of a radar truss for $t_{b}=t_{d}=4.8$ $\times 10^{-4} \mathrm{~m} t_{L}=2.4 \times 10^{-4} \mathrm{~m}$ and $L_{\text {tot }}=300 \mathrm{~m}$ in $(\mathrm{rad} / \mathrm{sec})$

\begin{tabular}{ccccc}
\hline Configuration 1 & Configuration 2 & PFC & Configuration 3 & PFC \\
\hline 26.098 & 26.432 & 1.279 & 26.779 & 2.609 \\
52.195 & 52.863 & 1.279 & 53.558 & 2.611 \\
78.293 & 79.295 & 1.279 & 80.337 & 2.610 \\
104.391 & 105.727 & 1.279 & 107.116 & 2.610 \\
130.488 & 132.159 & 1.280 & 133.895 & 2.611 \\
156.586 & 158.590 & 1.279 & 160.674 & 2.610 \\
182.684 & 185.022 & 1.279 & 187.453 & 2.610 \\
208.782 & 211.454 & 1.279 & 214.231 & 2.609 \\
234.879 & 237.886 & 1.280 & 241.010 & 2.610 \\
260.977 & 264.317 & 1.279 & 267.789 & 2.610 \\
287.075 & 290.749 & 1.279 & 294.568 & 2.610 \\
313.172 & 317.181 & 1.280 & 321.347 & 2.610 \\
339.270 & 343.613 & 1.280 & 348.126 & 2.610 \\
365.368 & 370.044 & 1.279 & 374.905 & 2.610 \\
391.465 & 396.476 & 1.280 & 401.684 & 2.610 \\
417.563 & 422.908 & 1.280 & 428.463 & 2.610 \\
443.661 & 449.340 & 1.280 & 455.242 & 2.610 \\
469.759 & 475.771 & 1.279 & 482.021 & 2.610 \\
495.856 & 502.203 & 1.280 & 508.800 & 2.610 \\
521.954 & 528.635 & 1.280 & 535.579 & 2.610 \\
\hline & & & &
\end{tabular}


Table 21

Bending natural frequencies of a radar truss for $t_{b}=4.8 \times$ $10^{-4} \mathrm{~m} t_{L}=t_{d}=2.4 \times 10^{-4} \mathrm{~m}$ and $L_{t o t}=300 \mathrm{~m}$ in $(\mathrm{rad} / \mathrm{sec})$

\begin{tabular}{ccccc}
\hline Configuration 1 & Configuration 2 & PFC & Configuration 3 & PFC \\
\hline 0.845 & 0.856 & 1.302 & 0.867 & 2.604 \\
2.326 & 2.356 & 1.290 & 2.387 & 2.623 \\
4.547 & 4.605 & 1.276 & 4.666 & 2.617 \\
7.486 & 7.582 & 1.282 & 7.681 & 2.605 \\
11.125 & 11.268 & 1.285 & 11.416 & 2.616 \\
15.443 & 15.641 & 1.282 & 15.846 & 2.610 \\
20.415 & 20.676 & 1.279 & 20.948 & 2.611 \\
26.012 & 26.345 & 1.280 & 26.691 & 2.610 \\
32.205 & 32.617 & 1.279 & 33.045 & 2.608 \\
38.962 & 39.460 & 1.278 & 39.978 & 2.608 \\
46.250 & 46.842 & 1.280 & 47.456 & 2.608 \\
54.037 & 54.728 & 1.279 & 55.446 & 2.608 \\
62.290 & 63.086 & 1.278 & 63.913 & 2.606 \\
70.975 & 71.882 & 1.278 & 72.825 & 2.607 \\
80.062 & 81.850 & 2.233 & 82.148 & 2.606 \\
89.519 & 90.663 & 1.278 & 91.852 & 2.606 \\
99.318 & 100.586 & 1.277 & 101.905 & 2.605 \\
109.429 & 110.827 & 1.277 & 112.279 & 2.604 \\
119.827 & 121.357 & 1.277 & 122.947 & 2.604 \\
130.485 & 132.151 & 1.277 & 133.882 & 2.603 \\
\hline
\end{tabular}

Table 22

Torsional natural frequencies of a radar truss for $t_{b}=4.8 \times$ $10^{-4} \mathrm{~m}_{L}=t_{d}=2.4 \times 10^{-4} \mathrm{~m}$ and $L_{t o t}=300 \mathrm{~m}$ in $(\mathrm{rad} / \mathrm{sec})$ \begin{tabular}{lllll}
\hline Configuration 1 & Configuration 2 & PFC & Configuration 3 & PFC \\
\hline
\end{tabular}

\begin{tabular}{rrrrr}
\hline 8.852 & 8.964 & 1.265 & 9.0813 & 2.590 \\
17.704 & 17.929 & 1.271 & 18.163 & 2.592 \\
26.556 & 26.893 & 1.269 & 27.244 & 2.590 \\
35.408 & 35.858 & 1.271 & 36.325 & 2.589 \\
44.260 & 44.822 & 1.270 & 45.406 & 2.589 \\
53.112 & 53.787 & 1.271 & 54.488 & 2.590 \\
61.964 & 62.751 & 1.270 & 63.569 & 2.590 \\
70.816 & 71.715 & 1.269 & 72.650 & 2.589 \\
79.668 & 80.680 & 1.270 & 81.732 & 2.590 \\
88.520 & 89.644 & 1.270 & 90.813 & 2.590 \\
97.372 & 98.609 & 1.270 & 99.894 & 2.590 \\
106.224 & 107.573 & 1.270 & 108.975 & 2.589 \\
115.076 & 116.538 & 1.270 & 118.057 & 2.590 \\
123.928 & 125.502 & 1.270 & 127.138 & 2.590 \\
132.780 & 134.467 & 1.270 & 136.219 & 2.590 \\
141.632 & 143.431 & 1.270 & 145.300 & 2.589 \\
150.484 & 152.395 & 1.269 & 154.382 & 2.590 \\
159.336 & 161.360 & 1.270 & 163.463 & 2.590 \\
168.188 & 170.324 & 1.270 & 172.544 & 2.590 \\
177.040 & 179.289 & 1.270 & 181.626 & 2.590 \\
\hline
\end{tabular}

Table 23

Longitudinal natural frequencies of a radar truss for $t_{b}=4.8$ $10^{-4} \mathrm{~m}_{L}=t_{d}=2.4 \times 10^{-4} \mathrm{~m}$ and $L_{t o t}=300 \mathrm{~m}$ in $(\mathrm{rad} / \mathrm{sec})$

\begin{tabular}{ccccc}
\hline Configuration 1 & Configuration 2 & PFC & Configuration 3 & PFC \\
\hline 29.104 & 29.476 & 1.278 & 29.864 & 2.611 \\
58.208 & 58.953 & 1.280 & 59.727 & 2.610 \\
87.312 & 88.429 & 1.279 & 89.591 & 2.610 \\
116.415 & 117.906 & 1.281 & 119.454 & 2.611 \\
145.519 & 147.382 & 1.280 & 149.318 & 2.611 \\
174.623 & 176.858 & 1.280 & 179.181 & 2.610 \\
203.727 & 206.335 & 1.280 & 209.045 & 2.610 \\
232.831 & 235.811 & 1.280 & 238.909 & 2.611 \\
261.935 & 265.287 & 1.280 & 268.772 & 2.610 \\
291.039 & 294.764 & 1.280 & 298.636 & 2.610 \\
320.143 & 324.240 & 1.280 & 328.499 & 2.610 \\
349.246 & 353.717 & 1.280 & 358.363 & 2.611 \\
378.350 & 383.193 & 1.280 & 388.226 & 2.610 \\
407.454 & 412.669 & 1.279 & 418.090 & 2.610 \\
436.558 & 442.146 & 1.280 & 447.954 & 2.610 \\
465.662 & 471.622 & 1.279 & 477.817 & 2.610 \\
494.766 & 501.098 & 1.279 & 507.681 & 2.610 \\
523.870 & 530.575 & 1.279 & 537.544 & 2.610 \\
552.973 & 560.051 & 1.280 & 567.408 & 2.610 \\
582.077 & 589.528 & 1.280 & 597.271 & 2.610 \\
\hline
\end{tabular}


the calculation of the frequencies. This results in having larger values compared to the Distributed Parameter Model (DPM) method. The difference between the FEA and the analytical DPM diminishes for larger lengths. Figure 7 indicates a noticeable increase of the frequencies when compared to Fig. 6 . This is due to the addition of the MFCs in the structure. Figure 8 presents the results for the bending frequencies for a truss with various longeron and diagonal thickness values and no actuators. As shown, increasing the thickness of the longeron members result in increasing the frequency values. This is also expected as these members contribute significantly to the bending stiffness of the truss compared to the diagonal and batten members. Therefore, increasing their thicknesses will have a larger impact on the overall stiffness of the truss compared to the overall mass which results in larger frequency values. This is in contrast to the diagonal members for which increasing their thickness results in a decrease of the frequencies as they add more mass than stiffness to the structure.

Tables 3 to 11 present the numerical results for bending, torsional, longitudinal frequencies and their PFC (Percentage Frequency Change) for various MFC configurations. The PFC compares the frequencies for configurations 2 and 3.

The results are shown for various lengths of the truss: 120 and 210 and 300 meters. It is shown that the frequencies change up to $3 \%$ as a result of the addition of the MFCs for these structures. In Tables 12 to 23 the frequency results for various thickness values are compared for the three MFC configurations. The effects of the MFC actuators are also clearly shown in these tables. A noticeable increase of the frequencies is shown due to the addition of MFCs. It is clear from these tables that the smaller the thicknesses of longerons and diagonals, the more important the effects of the MFCs on the natural frequencies become. This, indeed, emphasizes the importance of developing a modeling technique that accounts for the effects of the MFCs on the dynamics of inflatable and gossamer structures due to being ultra-light-weight and flexible.

\section{Conclusion}

An approximate beam-like dynamic model for an inflatable rigidized lattice structure with embedded MFC actuators was derived using a continuum modeling approach. A comparison of the natural frequencies of this model with those of finite element analysis demonstrated the precision of the continuum model and thus its usefulness in analyzing complex truss structures. The natural frequencies of lattice structures with two distributed actuator configurations, one with more actuators per member, were compared with the case of a structure with no integrated MFC actuation. The change in natural frequency as a result of adding the MFCs was shown to vary within 3\%, most significantly for the bending frequencies. These frequency changes are particularly important and must be considered when designing controllers for such lightweight structures. The developed model has the advantage of providing a closed form solution which provides the designer with great insight about the dominant physics and the underlying mathematics of the problem. In addition, closed form solutions are much faster; in the case for this structure the CPU usage was 180 times less than a MATLAB FEA solver. The numerical results are presented for various MFC configurations.

\section{Appendix A}

\section{Strain energy}

Taking spatial derivatives of the displacement field in Eq. (1), one can easily find the strain field for the fundamental truss element as follows $[5,7,8]$ :

$$
\begin{aligned}
& \varepsilon_{11}=\frac{\partial u_{1}}{\partial x_{1}}=\frac{\partial u_{1,0}}{\partial x_{1}}-x_{2} \frac{\partial \phi_{3}}{\partial x_{1}}+x_{3} \frac{\partial \phi_{2}}{\partial x_{1}}=\varepsilon_{1,0}-x_{2} \kappa_{2,0}+x_{3} \kappa_{3,0} \\
& \varepsilon_{22}=\frac{\partial u_{2}}{\partial x_{2}}=\varepsilon_{2,0} \\
& \varepsilon_{33}=\frac{\partial u_{3}}{\partial x_{3}}=\varepsilon_{3,0}
\end{aligned}
$$




$$
\begin{aligned}
\varepsilon_{12} & =\frac{1}{2}\left(\frac{\partial u_{1}}{\partial x_{2}}+\frac{\partial u_{2}}{\partial x_{1}}\right)=\frac{1}{2}\left(\left(\frac{\partial u_{2,0}}{\partial x_{1}}-\phi_{3}\right)+x_{2} \frac{\partial \varepsilon_{2,0}}{\partial x_{1}}+x_{3}\left[-\frac{\partial \phi_{1}}{\partial x_{1}}+\frac{1}{2}\left(2 \frac{\partial \varepsilon_{23,0}}{\partial x_{1}}\right)\right]\right) \\
& =\frac{1}{2}\left(2 \varepsilon_{12,0}+x_{2} \frac{\partial \varepsilon_{2,0}}{\partial x_{1}}+x_{3}\left[-\kappa_{1,0}+\frac{1}{2}\left(2 \frac{\partial \varepsilon_{23,0}}{\partial x_{1}}\right)\right]\right) \\
\varepsilon_{13} & =\frac{1}{2}\left(\frac{\partial u_{1}}{\partial x_{3}}+\frac{\partial u_{3}}{\partial x_{1}}\right)=\frac{1}{2}\left(\left(\frac{\partial u_{3,0}}{\partial x_{1}}+\phi_{2}\right)+x_{2}\left[\frac{\partial \phi_{1}}{\partial x_{1}}+\frac{1}{2}\left(2 \frac{\partial \varepsilon_{23,0}}{\partial x_{1}}\right)\right]+x_{3} \frac{\partial \varepsilon_{3,0}}{\partial x_{1}}\right)= \\
& =\frac{1}{2}\left(2 \varepsilon_{13,0}+x_{2}\left[\kappa_{1,0}+\frac{1}{2}\left(2 \frac{\partial \varepsilon_{23,0}}{\partial x_{1}}\right)\right]+x_{3} \frac{\partial \varepsilon_{3,0}}{\partial x_{1}}\right) \\
\varepsilon_{23} & =\frac{1}{2}\left(\left[-\phi_{1}+\frac{1}{2}\left(2 \varepsilon_{23,0}\right)\right]+\left[\phi_{1}+\frac{1}{2}\left(2 \varepsilon_{23,0}\right)\right]\right)=\varepsilon_{23,0}
\end{aligned}
$$

Here, the strain components $\varepsilon_{1,0}, \varepsilon_{2,0}, \varepsilon_{3,0}, \varepsilon_{12,0}, \varepsilon_{13,0}$ and $\varepsilon_{23,0}$ are the shear and extensional strain components evaluated at the center of a cross section of the truss element shown in Fig. 2. These strain components depend on $x_{1}$ coordinate of the cross section only. Also $\kappa_{1,0}, \kappa_{2,0}$ and $\kappa_{3,0}$ are twist and curvature components for the strain field and they are all dependent on $x_{1}$ only. The strain components in Eq. (A-1) can be projected along each of the bar members to find the extensional strain energy of each member. This can be done for all the 18 bar members shown in Fig. 2. The strain energy of the truss element can then be found using the following relation:

$$
U^{(k)}=\frac{1}{2} K^{(k)}\left(L^{(k)}\right)^{2}\left(\varepsilon^{(k)}\right)^{2}
$$

where $\varepsilon^{(k)}$ is the axial strain along the length of each bar member which is evaluated at the center of the member. As an example, the strain energy of all the diagonal members can be found as:

$$
\begin{aligned}
U_{d}= & \frac{L_{d}^{2} K_{d}}{2\left(L_{L}^{2}+L_{b}^{2}\right)^{2}}\left(\left(L_{L}^{2} \varepsilon_{11}^{(13)}-2 L_{L} L_{b} \varepsilon_{12}^{(13)}+L_{b}^{2} \varepsilon_{22}^{(13)}\right)^{2}+\left(L_{L}^{2} \varepsilon_{11}^{(14)}+2 L_{L} L_{b} \varepsilon_{12}^{(14)}+L_{b}^{2} \varepsilon_{22}^{(14)}\right)^{2}+\right. \\
& +\left(L_{L}^{2} \varepsilon_{11}^{(15)}+L_{L} L_{b} \varepsilon_{12}^{(15)}-\sqrt{3} L_{L} L_{b} \varepsilon_{13}^{(15)}+\frac{L_{b}^{2}}{4} \varepsilon_{22}^{(15)}-\frac{\sqrt{3} L_{b}^{2}}{2} \varepsilon_{23}^{(15)}+\frac{3 L_{b}^{2}}{4} \varepsilon_{33}^{(15)}\right)^{2} \\
& +\left(L_{L}^{2} \varepsilon_{11}^{(16)}-L_{L} L_{b} \varepsilon_{12}^{(16)}+\sqrt{3} L_{L} L_{b} \varepsilon_{13}^{(16)}+\frac{L_{b}^{2}}{4} \varepsilon_{22}^{(16)}-\frac{\sqrt{3} L_{b}^{2}}{2} \varepsilon_{23}^{(16)}+\frac{3 L_{b}^{2}}{4} \varepsilon_{33}^{(16)}\right)^{2} \\
& +\left(L_{L}^{2} \varepsilon_{11}^{(17)}+L_{L} L_{b} \varepsilon_{12}^{(17)}+\sqrt{3} L_{L} L_{b} \varepsilon_{13}^{(17)}+\frac{L_{b}^{2}}{4} \varepsilon_{22}^{(17)}+\frac{\sqrt{3} L_{b}^{2}}{2} \varepsilon_{23}^{(17)}+\frac{3 L_{b}^{2}}{4} \varepsilon_{33}^{(17)}\right)^{2} \\
& \left.+\left(L_{L}^{2} \varepsilon_{11}^{(18)}-L_{L} L_{b} \varepsilon_{12}^{(18)}-\sqrt{3} L_{L} L_{b} \varepsilon_{13}^{(18)}+\frac{L_{b}^{2}}{4} \varepsilon_{22}^{(18)}+\frac{\sqrt{3} L_{b}^{2}}{2} \varepsilon_{23}^{(18)}+\frac{3 L_{b}^{2}}{4} \varepsilon_{33}^{(18)}\right)^{2}\right)^{2}
\end{aligned}
$$

The strain energy of the longerons and battens can be found in a very similar manner and from there the strain energy of the fundamental truss element can be found using the following relation:

$$
U_{e}=U_{L}+U_{b}+U_{d}
$$

The above strain energy is expressed in terms of a total of 21 strain and curvature components and their gradients, i.e.,

$\varepsilon_{1,0}, \varepsilon_{2,0}, \varepsilon_{3,0}, \varepsilon_{12,0}, \varepsilon_{23,0}, \varepsilon_{13,0}, \kappa_{1,0}, \kappa_{2,0}, \kappa_{3,0}, \frac{\partial \varepsilon_{1,0}}{\partial x_{1}}, \frac{\partial \varepsilon_{2,0}}{\partial x_{1}}, \frac{\partial \varepsilon_{3,0}}{\partial x_{1}}, \frac{\partial \varepsilon_{13,0}}{\partial x_{1}}, \frac{\partial \varepsilon_{23,0}}{\partial x_{1}}, \frac{\partial \varepsilon_{12,0}}{\partial x_{1}}, \frac{\partial^{2} \varepsilon_{2,0}}{\partial x_{1}^{2}}$,

$\frac{\partial^{2} \varepsilon_{3,0}}{\partial x_{1}^{2}}, \frac{\partial^{2} \varepsilon_{23,0}}{\partial x_{1}^{2}}, \frac{\partial \kappa_{1,0}}{\partial x_{1}}, \frac{\partial \kappa_{2,0}}{\partial x_{1}}$, and $\frac{\partial \kappa_{3,0}}{\partial x_{1}}$ 
To find a beam equivalent of the structure, it is important that all these strain components be related to the strain components in a one-dimensional structure. Therefore, similar assumptions to those made in Refs. [5,7,8] should be made to find an equivalent reduced order model (1-dimensional). One of these assumptions includes the assumption of the local free deformations which indicates the condition that forces associated with local deformations should vanish. Since forces can be expressed in terms of the derivatives of the strain energy with respect to their strain components, this condition requires these derivatives vanish. Therefore,

$$
\begin{gathered}
\frac{\partial U_{e}}{\partial \frac{\partial \varepsilon_{1,0}}{\partial x_{1}}}=0 \Rightarrow 3\left(L_{d}^{2} K_{d} L_{L}^{5}+L_{L}^{2} K_{L} L_{L}\left(L_{b}^{2}+L_{L}^{2}\right)^{2}\right) \frac{\partial \varepsilon_{1,0}}{\partial x_{1}}-\sqrt{3} L_{d}^{2} K_{d} L_{b}^{2} L_{L}^{3} \kappa_{1,0}=0 \\
\frac{\partial U_{e}}{\partial \frac{\partial \kappa_{2,0}}{\partial x_{1}}}=0 \Rightarrow\left(L_{d}^{2} K_{d} L_{L}^{4}+4 L_{L}^{2} K_{L}\left(L_{b}^{2}+L_{L}^{2}\right)^{2}\right) \frac{\partial \kappa_{2,0}}{\partial x_{1}}+8 \sqrt{3} L_{d}^{2} K_{d} L_{L}^{2} \varepsilon_{13,0}=0 \\
\frac{\partial U_{e}}{\partial \frac{\partial \kappa_{3,0}}{\partial x_{1}}}=0 \Rightarrow\left(L_{d}^{2} K_{d} L_{L}^{4}+4 L_{L}^{2} K_{L}\left(L_{b}^{2}+L_{L}^{2}\right)^{2}\right) \frac{\partial \kappa_{3,0}}{\partial x_{1}}+8 \sqrt{3} L_{d}^{2} K_{d} L_{L}^{2} \varepsilon_{12,0}=0 \\
\frac{\partial U_{e}}{\partial \frac{\partial \varepsilon_{12,0}}{\partial x_{1}}}=0 \Rightarrow 3 L_{b} L_{L}^{2} \frac{\partial^{2} \varepsilon_{2,0}}{\partial x_{1}^{2}}-3 L_{b} L_{L}^{2} \frac{\partial^{2} \varepsilon_{3,0}}{\partial x_{1}^{2}}+4 L_{b}\left(\sqrt{3} L_{L}^{2} \frac{\partial^{2} \varepsilon_{23,0}}{\partial x_{1}^{2}}+6 \varepsilon_{2,0}-6 \varepsilon_{3,0}\right) \\
-8 L_{L}^{2}\left(6 \frac{\partial \varepsilon_{12,0}}{\partial x_{1}}+\sqrt{3} \kappa_{3,0}\right)=0
\end{gathered}
$$

$$
\begin{aligned}
\frac{\partial U_{e}}{\partial \frac{\partial \varepsilon_{13,0}}{\partial x_{1}}=}= & \Rightarrow \sqrt{3} L_{b} L_{L}^{2} \frac{\partial^{2} \varepsilon_{2,0}}{\partial x_{1}^{2}}-24 L_{b} \varepsilon_{23,0}-L_{L}^{2} \\
& \left(\sqrt{3} L_{b} \frac{\partial^{2} \varepsilon_{3,0}}{\partial x_{1}^{2}}+3 L_{b} \frac{\partial^{2} \varepsilon_{23,0}}{\partial x_{1}^{2}}+24 \frac{\partial \varepsilon_{13,0}}{\partial x_{1}}+4 \sqrt{3} \kappa_{2,0}\right)=0 \\
\frac{\partial U_{e}}{\partial \frac{\partial \kappa_{1,0}}{\partial x_{1}}=}= & \Rightarrow L_{L}^{2}\left(L_{b}^{2}\left(\sqrt{3} \frac{\partial^{2} \varepsilon_{2,0}}{\partial x_{1}^{2}}+\sqrt{3} \frac{\partial^{2} \varepsilon_{3,0}}{\partial x_{1}^{2}}-4 \frac{\partial \kappa_{1,0}}{\partial x_{1}}\right)+16 \sqrt{3} \varepsilon_{1,0}\right) \\
& +8 \sqrt{3} L_{b}^{2}\left(\varepsilon_{2,0}+\varepsilon_{3,0}\right)=0 \\
\frac{\partial U_{e}}{\partial \frac{\partial^{2} \varepsilon_{2,0}}{\partial x_{1}^{2}}=} & \Rightarrow L_{L}^{2}\left(13 L_{d}^{2} K_{d} L_{b}^{4}+72 L_{b}^{2} K_{b}\left(L_{b}^{2}+L_{L}^{2}\right)^{2}\right) \frac{\partial^{2} \varepsilon_{2,0}}{\partial x_{1}^{2}}+L_{L}^{2} \\
& \left(-L_{d}^{2} K_{d} L_{b}^{4}+24 L_{b}^{2} K_{b}\left(L_{b}^{2}+L_{L}^{2}\right)^{2}\right) \frac{\partial^{2} \varepsilon_{3,0}}{\partial x_{1}^{2}} \\
& -8\left(6 L_{d}^{2} K_{d} L_{b}^{3} L_{L}^{2} \frac{\partial \varepsilon_{12,0}}{\partial x_{1}}-6 L_{b}^{2} K_{b}\left(L_{b}^{2}+L_{L}^{2}\right)^{2}\left(3 \varepsilon_{2,0}+\varepsilon_{3,0}\right)\right. \\
& +L_{d}^{2} K_{d} L_{b}^{2}\left(-12 L_{L}^{2} \varepsilon_{1,0}+L_{b}^{2}\left(\sqrt{3} L_{L}^{2} \frac{\partial \kappa_{1,0}}{\partial x_{1}}-9 \varepsilon_{2,0}-3 \varepsilon_{3,0}+4 \sqrt{3} \varepsilon_{23,0}\right)\right. \\
& \left.\left.+L_{b} L_{L}^{2}\left(4 \sqrt{3} \frac{\partial \varepsilon_{13,0}}{\partial x_{1}}+2 \kappa_{2,0}+\sqrt{3} \kappa_{3,0}\right)\right)\right)
\end{aligned}
$$




$$
\begin{aligned}
\frac{\partial U_{e}}{\partial \frac{\partial^{2} \varepsilon_{3,0}}{\partial x_{1}^{2}}=} & \Rightarrow L_{L}^{2}\left(-L_{d}^{2} K_{d} L_{b}^{4}+24 L_{b}^{2} K_{b}\left(L_{b}^{2}+L_{L}^{2}\right)^{2}\right) \frac{\partial^{2} \varepsilon_{2,0}}{\partial x_{1}^{2}}+L_{L}^{2} \\
& \left(13 L_{d}^{2} K_{d} L_{b}^{4}+72 L_{b}^{2} K_{b}\left(L_{b}^{2}+L_{L}^{2}\right)^{2}\right) \frac{\partial^{2} \varepsilon_{3,0}}{\partial x_{1}^{2}} \\
& +8\left(6 L_{d}^{2} K_{d} L_{L}^{2} L_{b}^{3} \frac{\partial \varepsilon_{12,0}}{\partial x_{1}}+6 L_{b}^{2} K_{b}\left(L_{b}^{2}+L_{L}^{2}\right)^{2}\left(\varepsilon_{2,0}+\varepsilon_{3,0}\right)\right. \\
& +L_{d}^{2} K_{d} L_{b}^{2}\left(12 L_{L}^{2} \varepsilon_{1,0}+L_{b}^{2}\left(-\sqrt{3} L_{L}^{2} \frac{\partial \kappa_{1,0}}{\partial x_{1}}+3 \varepsilon_{2,0}+9 \varepsilon_{3,0}+4 \sqrt{3} \varepsilon_{23,0}\right)\right) \\
& \left.+L_{d}^{2} K_{d} L_{b}^{3} L_{L}^{2}\left(4 \sqrt{3} \frac{\partial \varepsilon_{13,0}}{\partial x_{1}}+2 \kappa_{2,0}+\sqrt{3} \kappa_{3,0}\right)\right) \\
\frac{\partial U_{e}}{\partial \frac{\partial^{2} \varepsilon_{23,0}}{\partial x_{1}^{2}}=} & 0 \Rightarrow L_{L}^{3}\left(7 L_{d}^{2} K_{d} L_{b}^{4}+24 L_{b}^{2} K_{b}\left(L_{b}^{2}+L_{L}^{2}\right)^{2}\right) \frac{\partial^{2} \varepsilon_{23,0}}{\partial x_{1}^{2}} \\
& +4 L_{L}\left(6\left(L_{d}^{2} K_{d} L_{b}^{4}+2 L_{b}^{2} K_{b}\left(L_{b}^{2}+L_{L}^{2}\right)^{2}\right) \varepsilon_{23,0}\right. \\
& \left.+L_{d}^{2} K_{d} L_{b}^{3}\left(2 \sqrt{3} L_{b}\left(\varepsilon_{2,0}-\varepsilon_{3,0}\right)+L_{L}^{2}\left(-4 \sqrt{3} \frac{\partial \varepsilon_{12,0}}{\partial x_{1}}+6 \frac{\partial \varepsilon_{13,0}}{\partial x_{1}}+\sqrt{3} \kappa_{2,0}-2 \kappa_{3,0}\right)\right)\right)
\end{aligned}
$$

Similarly to get a shear deformation type beam the following should hold $[5,7,8]$ :

$$
\begin{aligned}
& \frac{\partial U_{e}}{\partial \varepsilon_{2,0}}=0 \Rightarrow \\
& 9 L_{L}^{2}\left(L_{d}^{2} K_{d} L_{b}^{4}+2 L_{b}^{2} K_{b}\left(L_{b}^{2}+L_{L}^{2}\right)^{2}\right) \frac{\partial^{2} \varepsilon_{2,0}}{\partial x_{1}^{2}}+3 L_{L}^{2}\left(L_{d}^{2} K_{d} L_{b}^{4}+2 L_{b}^{2} K_{b}\left(L_{b}^{2}+L_{L}^{2}\right)^{2}\right) \frac{\partial^{2} \varepsilon_{3,0}}{\partial x_{1}^{2}} \\
& +24 L_{b}^{2} K_{b}\left(L_{L}^{2}+L_{b}^{2}\right)^{2}\left(3 \varepsilon_{2,0}+\varepsilon_{3,0}\right) \\
& +4 L_{d}^{2} K_{d} L_{b}^{2}\left(24 L_{L}^{2} \varepsilon_{1,0}+L_{b}^{2}\left(\sqrt{3} L_{L}^{2}\left(\frac{\partial^{2} \varepsilon_{23,0}}{\partial x_{1}^{2}}-2 \frac{\partial \kappa_{1,0}}{\partial x_{1}}\right)+6\left(3 \varepsilon_{2,0}+\varepsilon_{3,0}\right)\right)\right. \\
& \left.-2 L_{b} L_{L}^{2}\left(6 \frac{\partial \varepsilon_{12,0}}{\partial x_{1}}+\sqrt{3} \kappa_{3,0}\right)\right) \\
& \frac{\partial U_{e}}{\partial \varepsilon_{3,0}}=0 \Rightarrow 3 L_{L}^{2}\left(L_{d}^{2} K_{d} L_{b}^{4}+2 L_{b}^{2} K_{b}\left(L_{L}^{2}+L_{b}^{2}\right)^{2}\right) \frac{\partial^{2} \varepsilon_{2,0}}{\partial x_{1}^{2}}+9 L_{L}^{2}\left(L_{d}^{2} K_{d} L_{b}^{4}+2 L_{b}^{2} K_{b}\left(L_{b}^{2}+L_{L}^{2}\right)^{2}\right) \frac{\partial^{2} \varepsilon_{3,0}}{\partial x_{1}^{2}} \\
& +4 L_{d}^{2} K_{d} L_{b}^{2}\left(24 L_{L}^{2} \varepsilon_{1,0}+L_{b}^{2}\left(-\sqrt{3} L_{L}^{2}\left(\frac{\partial^{2} \varepsilon_{23,0}}{\partial x_{1}^{2}}+2 \frac{\partial \kappa_{1,0}}{\partial x_{1}}\right)+6\left(\varepsilon_{2,0}+3 \varepsilon_{3,0}\right)\right)\right. \\
& \left.+2 L_{b} L_{L}^{2}\left(6 \frac{\partial \varepsilon_{12,0}}{\partial x_{1}}+\sqrt{3} \kappa_{3,0}\right)+24 L_{b}^{2} K_{b}\left(L_{b}^{2}+L_{L}^{2}\right)^{2}\left(\varepsilon_{2,0}+3 \varepsilon_{3,0}\right)\right)=0 \\
& \frac{\partial U_{e}}{\partial \varepsilon_{23,0}}=0 \Rightarrow 6 L_{b}^{2} K_{b}\left(L_{b}^{2}+L_{L}^{2}\right)^{2}\left(L_{L}^{2} \frac{\partial^{2} \varepsilon_{23,0}}{\partial x_{1}^{2}}+4 \varepsilon_{23,0}\right) \\
& +L_{d}^{2} K_{d} L_{b}^{3}\left(-\sqrt{3} L_{b} L_{L}^{2} \frac{\partial^{2} \varepsilon_{2,0}}{\partial x_{1}^{2}}+24 L_{b} \varepsilon_{23,0}\right. \\
& \left.+L_{L}^{2}\left(\sqrt{3} L_{b} \frac{\partial^{2} \varepsilon_{3,0}}{\partial x_{1}^{2}}+3 L_{b} \frac{\partial^{2} \varepsilon_{23,0}}{\partial x_{1}^{2}}+24 \frac{\partial \varepsilon_{13,0}}{\partial x_{1}}+4 \sqrt{3} \kappa_{2,0}\right)\right)=0
\end{aligned}
$$


Finally, the compatibility condition requires that the strain components on the plane of the mid- cross section satisfy the following $[5,7,8]$ :

$$
\frac{\partial \varepsilon_{2,0}}{\partial x_{1}}=0, \frac{\partial \varepsilon_{3,0}}{\partial x_{1}}=0, \frac{\partial \varepsilon_{23,0}}{\partial x_{1}}=0
$$

Equations (A-5) to (A-16) along with the 3 equations in Eq. (A-17) give us a total of 15 equations to solve for the 15 of the 21 variables as follow:

$$
\begin{aligned}
& \frac{\partial \varepsilon_{12,0}}{\partial x_{1}}=-\frac{\kappa_{3,0}}{2 \sqrt{3}}, \quad \frac{\partial \kappa_{1,0}}{\partial x_{1}}=\frac{4 \sqrt{3} \varepsilon_{1,0}}{L_{b}^{2}}, \quad \frac{\partial \varepsilon_{13,0}}{\partial x_{1}}=-\frac{\kappa_{2,0}}{2 \sqrt{3}}, \quad \varepsilon_{2,0}=0, \quad \varepsilon_{3,0}=0, \quad \varepsilon_{23,0}=0, \\
& \frac{\partial \varepsilon_{1,0}}{\partial x_{1}}=\frac{K_{d} L_{b}^{2} \kappa_{1,0}}{\sqrt{3}\left(K_{d} L_{L}^{2}+K_{L}\left(L_{b}^{2}+L_{L}^{2}\right)\right)}, \frac{\partial \kappa_{3,0}}{\partial x_{1}}=-\frac{8 \sqrt{3} K_{d} \varepsilon_{12,0}}{K_{d} L_{L}^{2}+4 K_{L}\left(L_{b}^{2}+L_{L}^{2}\right)} \\
& \frac{\partial \kappa_{2,0}}{\partial x_{1}}=-\frac{8 \sqrt{3} K_{d} \varepsilon_{13,0}}{K_{d} L_{L}^{2}+4 K_{L}\left(L_{b}^{2}+L_{L}^{2}\right)}, \quad \frac{\partial^{2} \varepsilon_{2,0}}{\partial x_{1}^{2}}=0, \quad \frac{\partial^{2} \varepsilon_{23,0}}{\partial x_{1}^{2}}=0, \quad \frac{\partial^{2} \varepsilon_{3,0}}{\partial x_{1}^{2}}=0
\end{aligned}
$$

Therefore, we are left with 6 strain components $\varepsilon_{1,0}, \varepsilon_{12,0}, \quad \varepsilon_{13,0}, \kappa_{2,0}, \kappa_{3,0}$ and $\kappa_{1,0}$ which are similar to those in a continuum beam structure (One-dimensional). Finally, the strain energy for a truss element can be expressed in a quadratic form in terms of the strain values as:

$$
U_{e}=C_{1} \varepsilon_{1,0}^{2}+C_{2} \varepsilon_{12,0}^{2}+C_{3} \varepsilon_{13,0}^{2}+C_{4} \kappa_{2,0}^{2}+C_{5} \kappa_{3,0}^{2}+C_{6} \kappa_{1,0}^{2}
$$

where,

$$
\begin{aligned}
& C_{1}=3 L_{L}^{2} K_{L} \quad C_{2}=C_{3}=\frac{24 L_{L}^{2} L_{b}^{2} K_{L} K_{d}}{K_{d} L_{L}^{2}+4 K_{L} L_{d}^{2}} \\
& C_{4}=C_{5}=\frac{1}{2} L_{L}^{2} L_{b}^{2} K_{L} \quad C_{6}=\frac{L_{L}^{2} L_{b}^{4} K_{L} K_{d}}{4\left(K_{d} L_{L}^{2}+K_{L} L_{d}^{2}\right)}
\end{aligned}
$$

Recalling the definition of $\varepsilon_{1,0}, \quad \varepsilon_{12,0}, \quad \varepsilon_{13,0}, \kappa_{2,0}, \kappa_{3,0}$ and $\kappa_{1,0}$ we get:

$$
\begin{gathered}
\varepsilon_{1,0}=\frac{\partial u_{1,0}}{\partial x_{1}}, \varepsilon_{12,0}=\frac{1}{2}\left(\frac{\partial u_{2,0}}{\partial x_{1}}-\phi_{3}\right), \varepsilon_{13,0}=\frac{1}{2}\left(\frac{\partial u_{3,0}}{\partial x_{1}}+\phi_{2}\right), \\
\kappa_{2,0}=\frac{\partial \phi_{3}}{\partial x_{1}}, \kappa_{3,0}=\frac{\partial \phi_{2}}{\partial x_{1}}, \kappa_{1,0}=\frac{\partial \phi_{1}}{\partial x_{1}}
\end{gathered}
$$

Equations (A-19), (A-20) and (A-21) result in:

$$
\begin{aligned}
U_{e}= & 3 L_{L}^{2} K_{L}\left(\frac{\partial u_{1,0}}{\partial x_{1}}\right)^{2}+\frac{6 L_{L}^{2} L_{b}^{2} K_{L} K_{d}}{K_{d} L_{L}^{2}+4 K_{L} L_{d}^{2}}\left(\frac{\partial u_{2,0}}{\partial x_{1}}-\phi_{3}\right)^{2}+\frac{6 L_{L}^{2} L_{b}^{2} K_{L} K_{d}}{K_{d} L_{L}^{2}+4 K_{L} L_{d}^{2}}\left(\frac{\partial u_{3,0}}{\partial x_{1}}+\phi_{2}\right)^{2} \\
& +\frac{1}{2} L_{L}^{2} L_{b}^{2} K_{L}\left(\frac{\partial \phi_{3}}{\partial x_{1}}\right)^{2}+\frac{1}{2} L_{L}^{2} K_{L} L_{b}^{2}\left(\frac{\partial \phi_{2}}{\partial x_{1}}\right)^{2}+\frac{L_{L}^{2} L_{b}^{4} K_{L} K_{d}}{4\left(K_{d} L_{L}^{2}+K_{L} L_{d}^{2}\right)}\left(\frac{\partial \phi_{1}}{\partial x_{1}}\right)^{2}
\end{aligned}
$$

\section{Kinetic energy}

The next step to help find PDEs of motion of the equivalent DPM is to calculate the kinetic energy of the fundamental truss element. It can be shown very simply that the kinetic energy of a bar member can be written in the form:

$$
T=\frac{1}{6} \rho A l\left(V_{1 x}^{2}+V_{1 y}^{2}+V_{1 z}^{2}+V_{2 x}^{2}+V_{2 y}^{2}+V_{2 z}^{2}+V_{1 x} V_{2 x}+V_{1 y} V_{2 y}+V_{1 z} V_{2 z}\right)
$$

where the first subscript denotes each of the two end nodes of a bar and the second subscript denotes the projection of the velocity along the axis of the coordinate system. Using Eqs (1) and (A-23), we can easily find the kinetic energy of the truss element in the following form: 


$$
\begin{aligned}
T_{e}= & m_{1}\left(\left(\frac{\partial u_{1,0}}{\partial t}\right)^{2}+\left(\frac{\partial u_{2,0}}{\partial t}\right)^{2}+\left(\frac{\partial u_{3,0}}{\partial t}\right)^{2}\right) \\
& +m_{2}\left(2\left(\frac{\partial \phi_{1,0}}{\partial t}\right)^{2}+\left(\frac{\partial \phi_{2,0}}{\partial t}\right)^{2}+\left(\frac{\partial \phi_{3,0}}{\partial t}\right)^{2}\right)
\end{aligned}
$$

where,

$$
\begin{aligned}
& m_{1}=3\left(\rho_{b} A_{b} L_{b}+\rho_{L} A_{L} L_{L}+\rho_{d} A_{d} L_{d}\right) \\
& m_{2}=\frac{L_{b}^{2}\left(\rho_{b} A_{b} L_{b}+2 \rho_{L} A_{L} L_{L}+\rho_{d} A_{d} L_{d}\right)}{4}
\end{aligned}
$$

These expressions are used to derive the PDE of motion for the overall structure.

\section{References}

[1] C. Jenkins, editor, Recent Advances in Gossamer Spacecraft, volume 212 of Progress in Astonautics and Aeronautics. AIAA, Reston, VA, 2006.

[2] C. Jenkins, editor, Gossamer Spacecraft: Membrane and Inflatable Structures Technology for Space Applications, volume 191 of Progress in Astonautics and Aeronautics. AIAA, Reston, VA, 2001.

[3] E.J. Ruggiero and D.J. Inman, Gossamer spacecraft: recent trends in design, analysis, experimentation, and control, Journal of Spacecraft and Rockets 43 (2006), 10-24.

[4] E.J. Ruggiero, A. Jha, G. Park and D.J. Inman, A literature review of ultra-light and inflated toroidal satellite components, The Shock and Vibration Digest 35 (2003), 171-181.

[5] A.K. Noor, M.S. Anderson and W.H. Greene, Continuum models for beam- and platelike lattice structures, AIAA Journal 16 (1978), 1219-1228.

[6] A.K. Noor, Thermal stress analysis of double-layered grids, Journal of the Structural Division, ASCE 104 (1978), 1-262.

[7] A. Salehian, E.M. Cliff and D.J. Inman, Continuum modeling of an innovative space-based radar antenna truss, ASCE Journal of Aerospace Engineering 19 (2006), 227-240.

[8] A. Salehian, T.M. Seigler and D.J. Inman, Dynamic effects of a radar panel mounted on a truss satellite, AIAA Journal 45 (2007), 1642-1654.

[9] A. Salehian and D.J. Inman, Micro-polar Continuous Modeling and Frequency Response Validation of a Lattice Structure, ASME Journal of Vibration and Acoustics 132 (2010), 1-7.

[10] A.K. Noor and C.M. Andersen, Analysis of Beamlike Lattice Trusses, Computer Methods in Applied Mechanics nics and Engineering 20(1) (1979), 53-70.

[11] A.K. Noor and W.C. Russell, Anisotropic Continuum Models for Beamlike Lattice Trusses, Computer Methods in Applied Mechanics and Engineering 57(3) (1986), 257-277.

[12] M.S. Lake and E.C. Kalng, Generation and Comparison of Globally Isotropic Space-Filling Truss Structures, AIAA Journal 30(5) (1992), 1416-1424.

[13] C.T. Sun and B.J. Kim, Continuum Modeling of Periodic Truss Structures, Damage Mechanics and Continuum Modeling, N. Stubbs and D. Krajcinovic, eds, American Society of Civil Engineers, New York, 1985, pp. 57-71.

[14] U. Lee, Dynamic Continuum Plate Representations of Large Thin Lattice Structures, AIAA Journal 31(9) (1993), $1734-1736$.

[15] U. Lee, Equivalent Continuum Representation of Lattice Beams: Spectral Element Approach, Engineering Structures 20(7) (1998), 587-592.

[16] B. Burgart and P. Cartraud, Continuum Modeling of Beamlike Lattice Trusses Using Averaging Method, Computers and Structures 73(1-5) (1999), 267-279.

[17] H. Tollenaere and D. Caillerie, Continuous Modeling of Lattice Structures by Homogenization, Advances in Engineering Software 29(7) (1989), 699-705.

[18] G. Park, K. Kim and D.J. Inman, Integration of smart materials into dynamics and control of inflatable space structures, Journal of Intelligent Material Systems and Structures 12 (2001), 423-433.

[19] H.A. Sodano, G. Park and D.J. Inman, An investigation into the performance of macro-fiber composites for sensing and structural vibration applications, Mechanical Systems \& Signal Processing 18 (2004), 683-697.

[20] A. Jha and D. Inman, Sliding mode Control of a Gossamer Structure Using Smart Materials, Journal of Vibration \& Control 10 (2004), 1199-1220.

[21] P.A. Tarazaga, D.J. Inman and W.K. Wilkie, Control of a space rigidizable boom using macro-fiber composite actuators, Journal of Vibration and Control 13 (2007), 935-950.

[22] J.M. Renno and D.J. Inman, Experimentally validated model of a membrane strip with multiple actuators, Journal of Spacecraft and Rockets 44 (2007), 1140-1151.

[23] A. Salehian and D.J. Inman, Dynamic Analysis of a Lattice Structure by Homogenization: Experimental Validation, Journal of Sound and Vibration 316(1-5) (2008), 180-197.

[24] D.J. Inman, Finite Element Method (3rd Ed.), Engineering Vibrations, Prentice-Hall, Inc., Upper Saddle River, New Jersey, (2008), 576-578. 

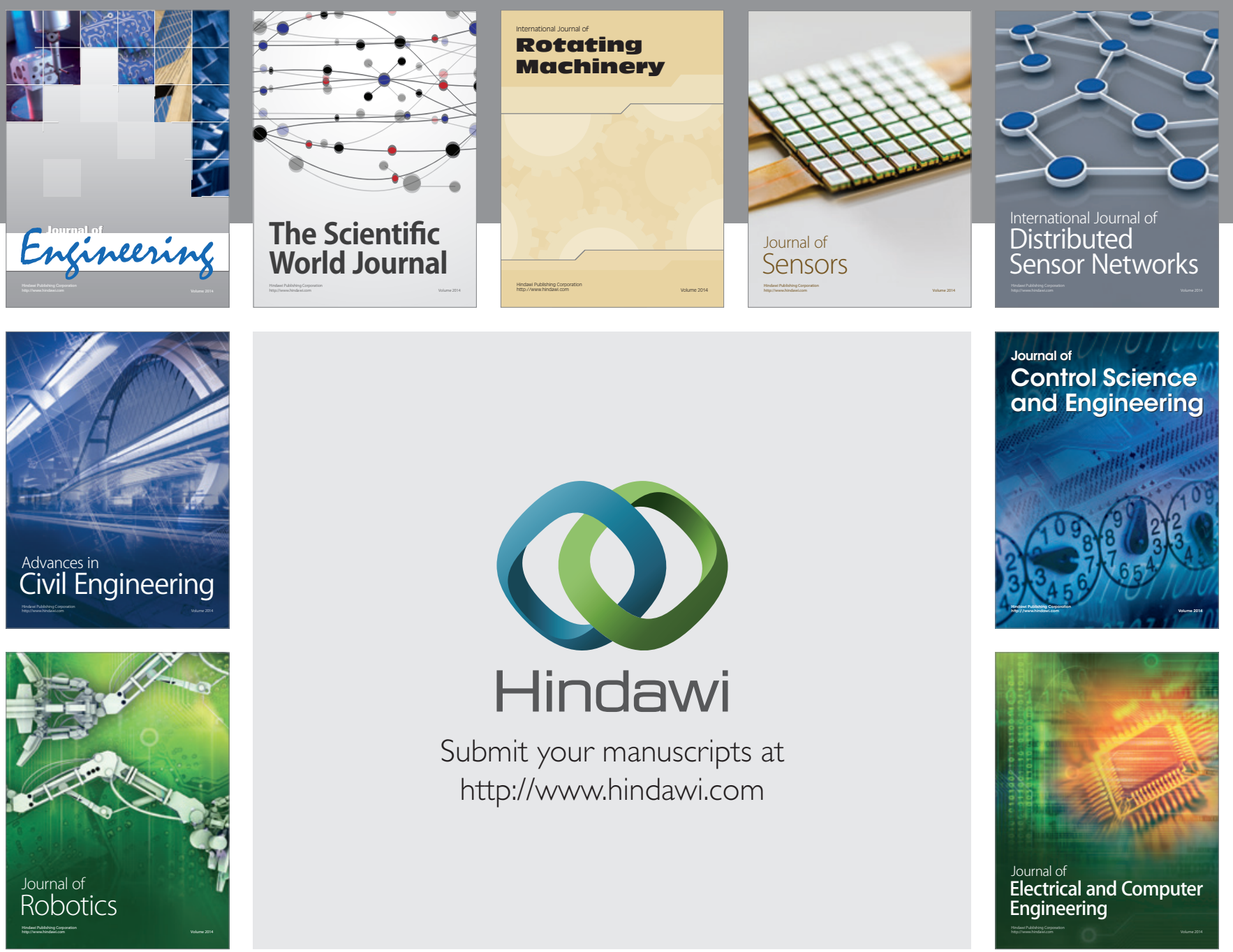

Submit your manuscripts at

http://www.hindawi.com
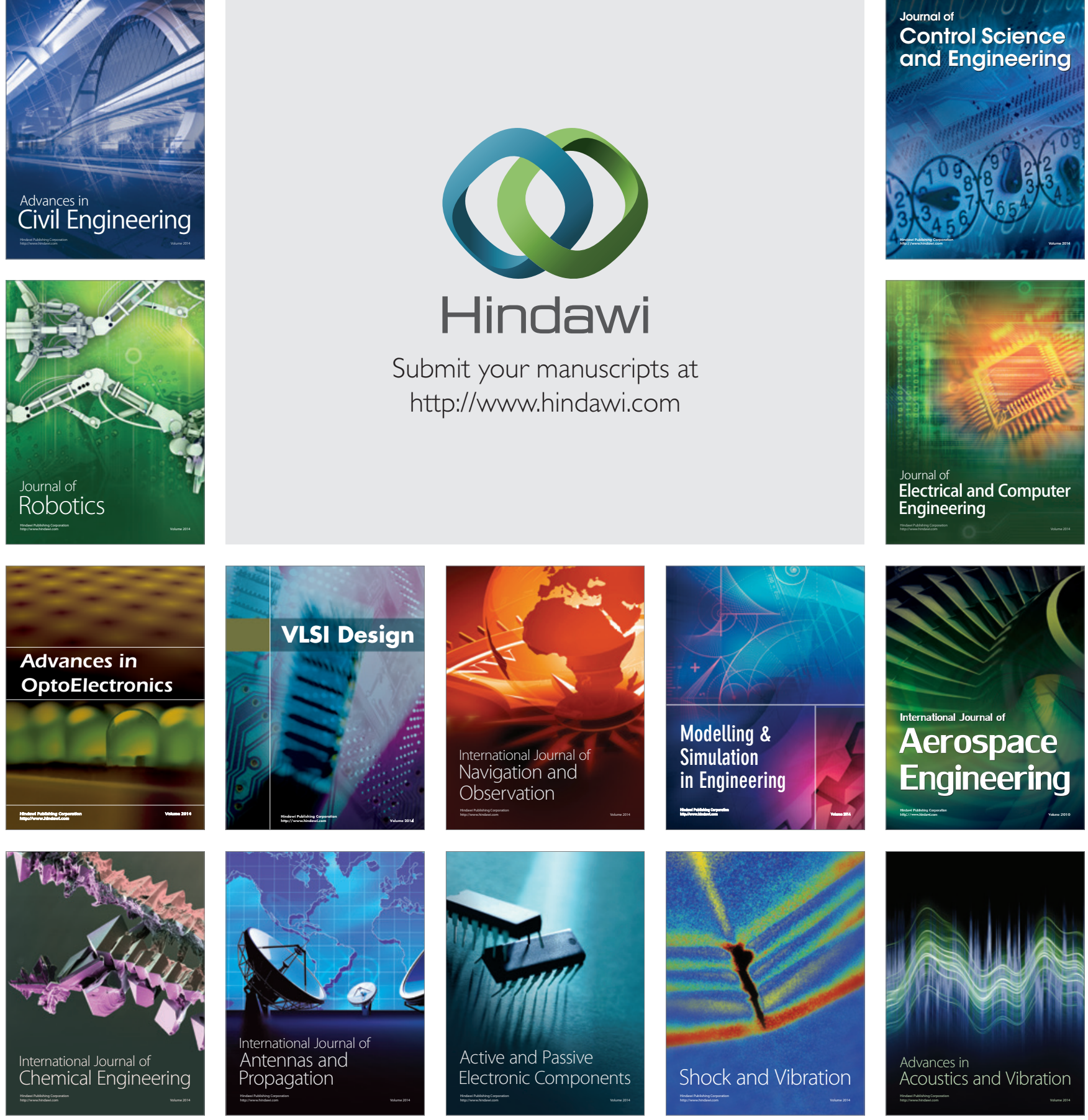